\title{
Design and Characterization
} of a

\section{Hypervelocity Expansion Tube Facility}

\author{
A. Dufrene* and M. Sharma ${ }^{\dagger}$ and J. M. Austin ${ }^{\ddagger}$ \\ Department of Aerospace Engineering, University of Illinois, Urbana-Champaign, 61801
}

\begin{abstract}
We report on the design and characterization of a $152 \mathrm{~mm}$ diameter expansion tube capable of accessing a range of high enthalpy test conditions with Mach numbers up to 7.1 for aerodynamic studies. Expansion tubes have the potential to offer a wide range of test flow conditions as gas acceleration is achieved through interaction with an unsteady expansion wave rather than expansion through a fixed area ratio nozzle. However, the range of test flow conditions is in practice limited by a number of considerations such as short test time and large amplitude flow disturbances. We present a generalized design strategy for small-scale expansion tubes. As a starting point, ideal gas dynamic calculations for optimal facility design to maximize test time at a given Mach number test condition are presented, together with a correction for the expansion head reflection through a non-simple region. A compilation of practical limitations that have been identified for expansion tube facilities such as diaphragm rupture and flow disturbance minimization is then used to map out a functional design parameter space. Experimentally, a range of test conditions have been verified through pitot pressure measurements and analysis of schlieren images of flow over simple geometries. To date there has been good agreement between theoretical and experimental results.
\end{abstract}

\section{Introduction}

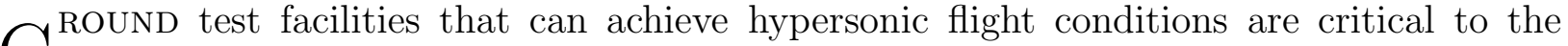
Gfurther development of re-entry vehicles and planetary probes for space exploration, as well as hypersonic air-breathing propulsion systems. The high stagnation enthalpy conditions necessary to study chemical and thermal real gas effects in hypervelocity flight can currently only be achieved in impulse facilities. ${ }^{1,2}$ Existing impulse hypervelocity facilities include large scale reflected shock tunnels such as T5 at Caltech, ${ }^{3}$ LENS at Calspan, ${ }^{4}$ and expansion

*Former Graduate Student, Department of Aerospace Engineering, University of Illinois, Professional Member AIAA.

${ }^{\dagger}$ Graduate Student, Department of Aerospace Engineering, University of Illinois

${ }^{\ddagger}$ Assistant Professor, Department of Aerospace Engineering, University of Illinois, Member AIAA 
tube facilities such as HYPULSE at GASL $^{5}$ and the X series facilities at the University of Queensland, Australia. ${ }^{6}$ An $89 \mathrm{~mm}$ diameter expansion tube facility operating with Mach numbers of 2.40 to 4.66 for scramjet combustion studies was recently built at Stanford. ${ }^{7}$ The smaller scale of this facility avoids the longer test preparation times needed in some of the larger scale tunnels. In the present paper, we report on the design and characterization of a new $152 \mathrm{~mm}$ diameter small-scale expansion tube facility that may be used for high speed aerodynamic studies with Mach numbers up to 7.1.

An expansion tube has several advantages and disadvantages in comparison with the more common reflected shock tunnel. ${ }^{1,2}$ In an expansion tunnel, the initial shock interacts with a contact surface generating an unsteady expansion that further accelerates the test gas (see Section II). Theoretically, a wide range of test conditions with decreased free stream dissociation can be achieved by varying initial pressures. ${ }^{8,9}$ In contrast, in a shock tunnel, chemical and vibrational freezing commonly occur in the nozzle, and the Mach number of the test gas can only be varied by exchanging the nozzles. However, expansion tubes tend to have shorter test times and increased boundary layer effects as the test condition is some distance downstream of the initial shock. In practice, expansion tube operating ranges have been limited by secondary diaphragm considerations and the appearance of large amplitude test flow disturbances.

We present a design procedure for small-scale expansion tubes. Ideal test condition calculations based on unsteady gas dynamics are first presented with particular focus on the important problem of short test times. The expansion tube test time is terminated by the arrival of the primary expansion wave from the driver, the tail of the secondary expansion wave or the leading characteristic of the reflected secondary expansion. In the latter case, we find the interaction of the two expansion waves significantly delays the arrival of the leading characteristic, and accounting for this effect may increase the theoretical test time by as much as $35 \%$.

An analytical expression based on the arrival time of the first reflected characteristic through the interaction of two expansion waves is presented. Optimal facility section lengths for maximizing test time at a given Mach number condition can be then calculated. In practice, the flow is significantly affected by the presence of the secondary diaphragm and nonideal diaphragm rupture may limit the range of useful test conditions. ${ }^{10,11}$ The light secondary diaphram may produce a reflected shock wave, have a finite opening time that reduces available test time, cause pressure disturbances, ${ }^{12}$ and produce fragments that affect test flow quality. We experimentally investigate optimizing the secondary diaphragm material, thickness, and burst mechanism for the initial pressures used in an expansion tube facility.

The potential for expansion tube facilities to access a very large range of test conditions was found to be limited, in practice, by the appearance of significant test flow disturbances as was seen in the NASA Langley expansion tube. ${ }^{9}$ Paull and Stalker ${ }^{13}$ found that at small expansion ratios (low enthalpy conditions), test gas disturbances result from the penetration of acoustic waves from the driver gas, which are focused into a narrow frequency band on transmission through the expansion. At high expansion ratios (high enthalpy conditions), an increased driver-gas sound speed ratio reduces the penetration of disturbances into the test gas. We apply Paull and Stalker's ${ }^{13}$ criteria for disturbance minimization together with other practical design considerations, to identify a useful parameter space for the operation of a small-scale expansion tube facility. 
The above procedure was used to design the $152 \mathrm{~mm}$ inner diameter hypervelocity expansion tube (HET) at the University of Illinois. Experimentally, a range of test conditions up to Mach 7.1 with suitable core flow size have been verified in this facility through pitot pressure measurements and the analysis of schlieren images of flow over simple geometries. Experimental and theoretical calulations are compared for a range of test conditions.

\section{Gas dynamic processes in the expansion tube}

The flow processes in an expansion tube are complex. Nevertheless, simple one-dimensional, inviscid, unsteady wave interactions may be used to calculate the ideal test conditions including thermodynamic state, flow velocity, and test time as a starting point for facility design.

\section{A. Calculation of thermodynamic state}

The expansion tube consists of three sections (a driver, driven and expansion section) separated by two diaphragms. A sample $x$ - $t$ diagram for a Mach 5.1 test flow is shown in Figure 1. The driver and driven sections operate as a standard shock tube. The strength of the primary left-facing expansion wave is dependent on the initial pressures, but it is in general sufficiently strong to accelerate the gas past sonic velocities. The head of the primary expansion wave will reflect from the upstream end of the tube and the tail will trail the primary contact surface.

The secondary diaphragm, assumed in this initial theoretical analysis to be thin and breaking instantaneously upon arrival of the incident shock, is represented as a contact surface. Pressure-velocity $(p-u)$ polars are used to calculate states 6 and 7 (see Figure 1) resulting from incident shock-contact surface interaction. A shock is transmitted and the expansion wave between regions 2 and 7 can be either right-facing or left-facing. From the $p$ - $u$ plane in Figure 2, it is apparent that the right-facing wave has no solution, so the wave is a left-facing expansion. The head and tail of the expansion have positive slopes as the flow in state 2 is supersonic and to the right. State 7 is the test gas that has been further accelerated beyond the post-shock state 2 by the unsteady secondary expansion.

The secondary expansion wave will interact with the contact surface between states 2 and 3 , resulting in reflected and transmitted waves. Examining the $p$ - $u$ diagram shows the reflected wave is also an expansion. Thus, a non-simple region is created where the two expansion waves interact.

\section{B. Test time calculation}

Test time varies with the initial conditions of the expansion tube, and can be estimated from an $x$ - $t$ diagram. The test time begins with the arrival of the state $6-7$ contact surface, and it is terminated by the arrival of either i) the head of the reflected secondary expansion wave, or ii) the tail of the secondary expansion wave, or iii) the arrival of the primary expansion from the driver, which ever occurs first. We consider cases i) and ii) first. For these two cases, the maximum test time corresponds to the case in which the reflected head and the tail of the secondary expansion fan arrive at the end of the tube at the same time.

The interaction of the incident and reflected secondary expansion waves creates a non- 


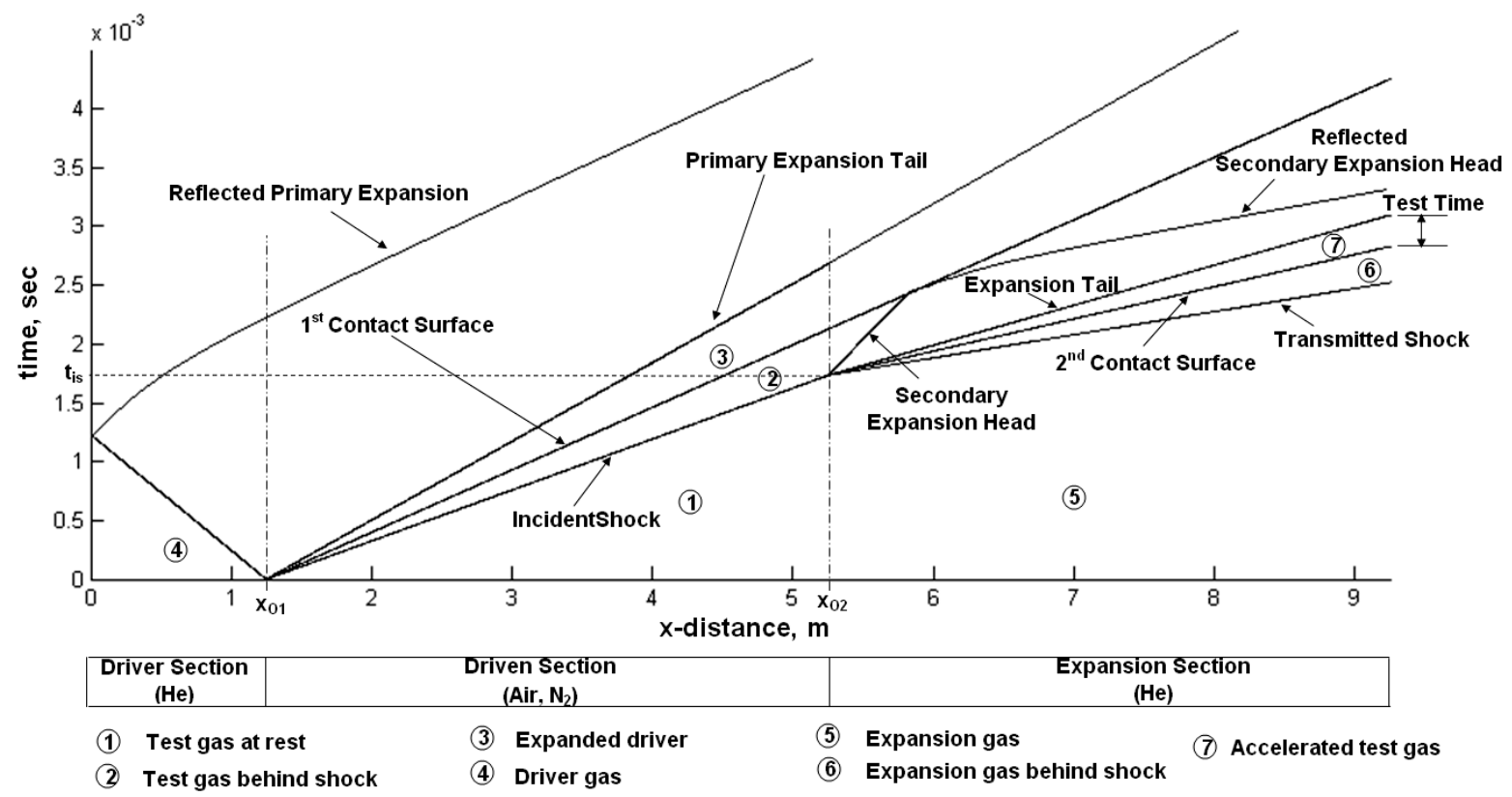

Figure 1. Sample $x$ - $t$ diagram of the calculated gas dynamic processes for a Mach 5.1 test flow in the HET facility. $\left(p_{4}=6250 \mathrm{kPa}, p_{1}=1.1 \mathrm{kPa}\right.$ and $\left.p_{5}=0.1 \mathrm{kPa}\right)$. The driver and expansion sections are filled with helium and the driven section with air. Calculations assume perfect gas.

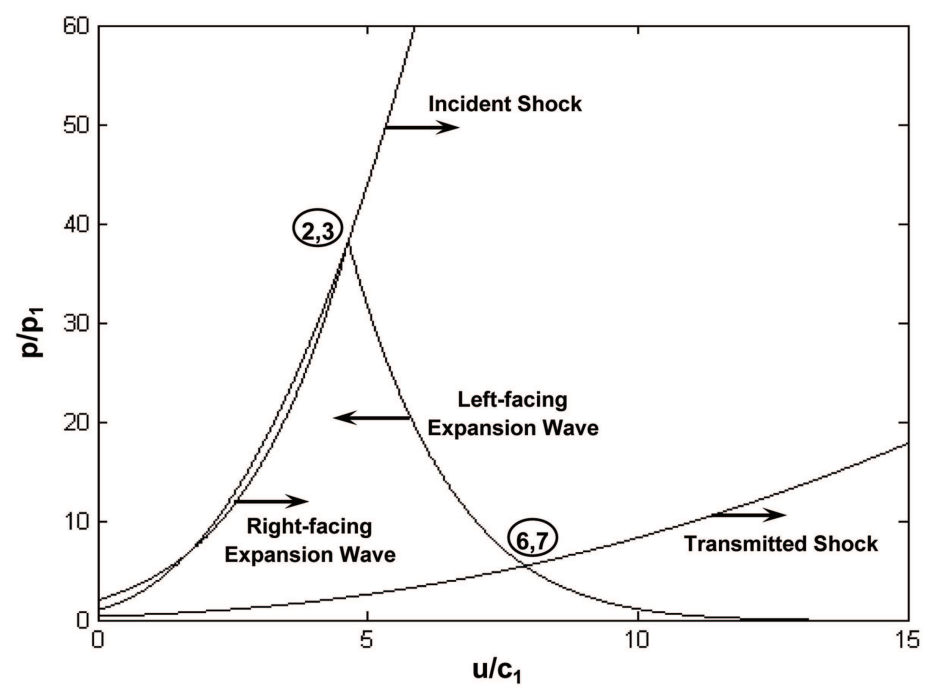

Figure 2. Normalized pressure and velocity polar calculation for incident shock interaction with secondary diaphragm represented as a contact surface. The calculations are performed for the same conditions as Figure 1. 
simple region. We may use the method of characteristics to solve for the trajectory of the leading characteristic of the reflected expansion as it passes through the incident expansion. A more detailed description is given in Wintenberger et al. ${ }^{14}$ Assuming an isentropic expansion, an ordinary differential equation for the passage of the first reflected characteristic through a left-facing expansion can be derived in terms of a similarity variable $\eta=\frac{x(t)}{c_{2} t}$ :

$$
\frac{d \eta}{d t}+\frac{2\left(\gamma_{2}-1\right)}{\gamma_{2}+1}\left(\eta-\frac{u_{2}}{c_{2}}-\frac{2}{\gamma_{2}-1}\right)=0
$$

which has the solution:

$$
\eta(t)=\frac{u_{2}}{c_{2}}+\frac{2}{\gamma_{2}-1}\left[1-\frac{\gamma_{2}+1}{2}\left(\frac{t}{\Delta t_{\text {int }}}\right)^{\frac{2\left(1-\gamma_{2}\right)}{\gamma_{2}+1}}\right]
$$

where $\Delta t_{\text {int }}$ is the time between the arrival of the initial shock at the secondary diaphragm location $x_{02}$ and the intersection between the first contact surface and the reflected expansion head as indicated in Figure 3 (the origin of Figure 3 is $t_{i s}$ and $x_{02}$ in Figure 1), $u_{2}$ is the gas velocity, $c_{2}$ is the gas sound speed and $\gamma_{2}$ is the specific gas ratio, which is assumed to be constant. Subscripts refer to the state as shown in Figure 1. The similarity solution must be referenced from the arrival of the initial shock at the secondary diaphragm location $x_{02}$. Figure 3 also shows the location of the leading characteristic of the reflected expansion with and without the correction for the interaction with the incident expansion. It was found that there was an error of up to thirty-five percent in calculating the test time for the reflected head limiting case when this correction is neglected. Table 1 presents some sample theoretical run conditions where this correction is important. The time $t_{\text {exit }}$ which corresponds to the intersection between the expansion tail and the reflected expansion head in the $x$ - $t$ plane can then be found from Equation 2 as:

$$
t_{\text {exit }}=\Delta t_{\text {int }}\left[\left(\frac{c_{7}}{c_{2}}\right)^{\frac{\gamma_{2}+1}{2\left(1-\gamma_{2}\right)}}-1\right]
$$

Figure 3 illustrates the situation where the test time is limited by the reflected expansion head, whereas Figure 1 is an example of an expansion tail limited solution. The maximum test time will occur when both of these waves arrive simultaneously at the end of the expansion section. Using the notation of Figure 3, the maximum test time $t_{\text {test,max }}$ would then be given as:

$$
t_{\text {test }, \text { max }}=\Delta t_{\text {int }}+t_{\text {exit }}-\Delta t_{2 c s}
$$

where $\Delta t_{2 c s}$ is defined in Figure 3 as the time difference between the second contact surface arrival at the tube end and the initial shock arrival at the secondary diaphragm. The optimal length of the facility expansion section for maximum test time $L_{\text {expansion }}$ can then be selected:

$$
L_{\text {expansion }}=\left(u_{7}-c_{7}\right)\left(t_{\text {exit }}+\Delta t_{\text {int }}\right)
$$

The optimal expansion section length as a function of the expansion-driven initial presssure ratio $\frac{p_{5}}{p_{1}}$ is shown in Figure 4 for different $\frac{p_{4}}{p_{1}}$ values. Lower $\frac{p_{5}}{p_{1}}$ values result in higher test flow Mach numbers and require longer expansion sections for maximum test time. It can be seen that the optimal expansion length parameter is insensitive to the driven-driver initial 


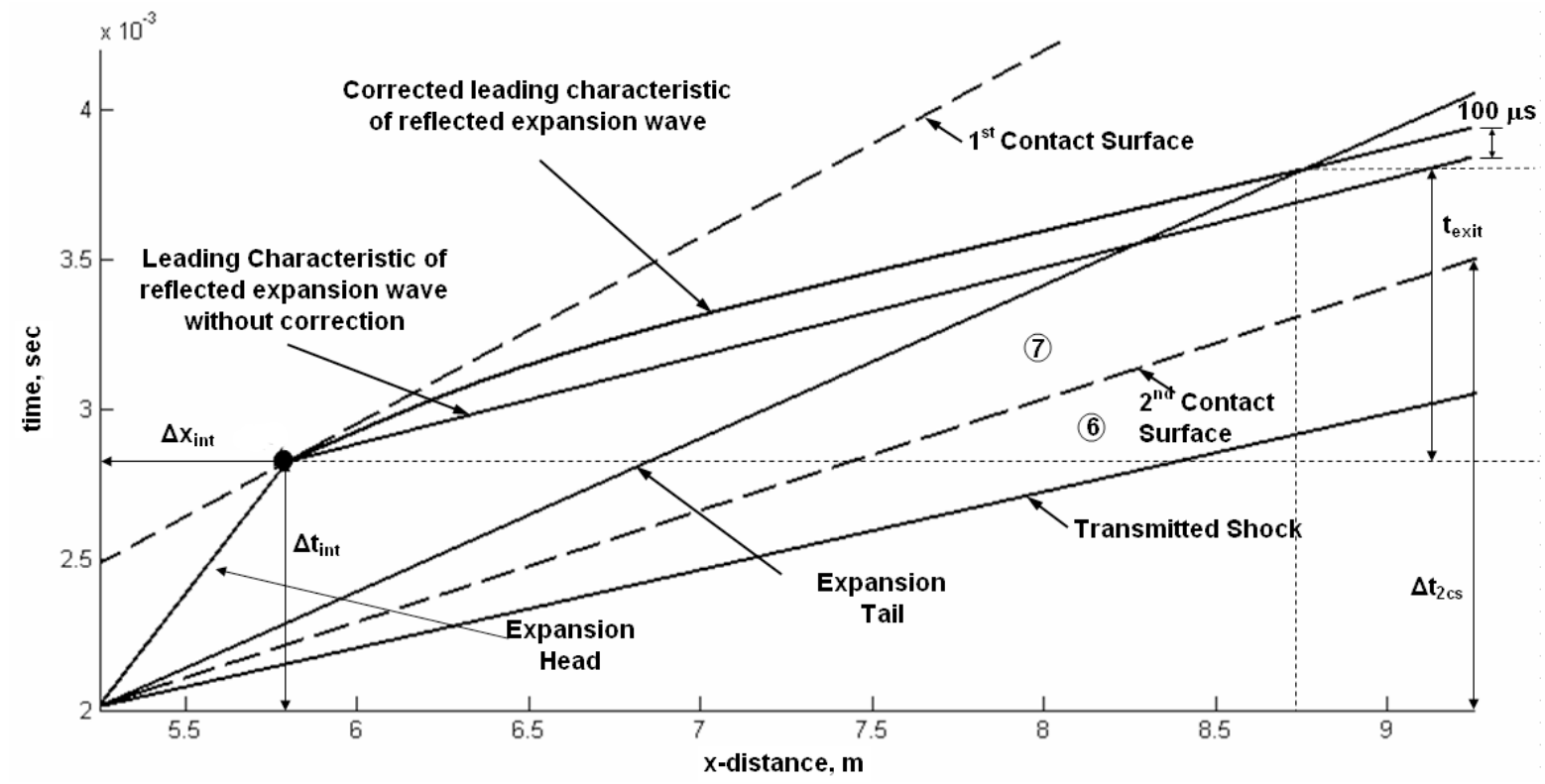

Figure 3. Detail from $x-t$ diagram from secondary diaphragm showing effect of the reflected wave correction for the interaction with the incident expansion wave. The origin of the $\mathrm{x}$-axis is the arrival of the incident shock at the secondary diaphragm station, $x_{02}=5.18 \mathrm{~m}$. The calculations are performed for the same conditions as Figure 1.

Table 1. Sample theoretical test conditions for expansion tube operation.

\begin{tabular}{|c|c|c|c|c|}
\hline & Condition 1 & Condition 2 & Condition 3 & Condition 4 \\
\hline Mach Number & 7.27 & 5.21 & 4.00 & 3.60 \\
\hline Static temperature, K & 588 & 1228 & 1392 & 930 \\
\hline Static pressure, $\mathrm{kPa}$ & 2.92 & 3.10 & 9.67 & 12.67 \\
\hline Velocity, m/s & 3540 & 3660 & 3000 & 2210 \\
\hline Density, $\mathrm{kg} / \mathrm{m}^{3}$ & 0.017 & 0.008 & 0.024 & 0.047 \\
\hline Test Time, $\mu \mathrm{s}$ & - & - & 335 & 447 \\
\hline Corrected Test Time $^{1}, \mu \mathrm{s}$ & 180 & 267 & 419 & 576 \\
\hline \multicolumn{5}{|l|}{ Initial Pressures, $k P a$} \\
\hline Driver Section & 6250 & 6250 & 5000 & 1250 \\
\hline Driven Section & 6.0 & 1.1 & 1.65 & 3.0 \\
\hline Expansion Section & 0.1 & 0.1 & 0.45 & 1.0 \\
\hline
\end{tabular}

1. The correction to the test time due to expansion wave interaction only applies when test time is terminated by arrival of the reflected expansion head (conditions 3 and 4). 
pressure ratio, $\frac{p_{4}}{p_{1}}$. The horizontal line in Figure 4 denotes the expansion section length selected for the current HET design. Solutions which fall below this line correspond to the test time being limited by the reflected secondary expansion head and those above it are restricted by the secondary expansion tail.

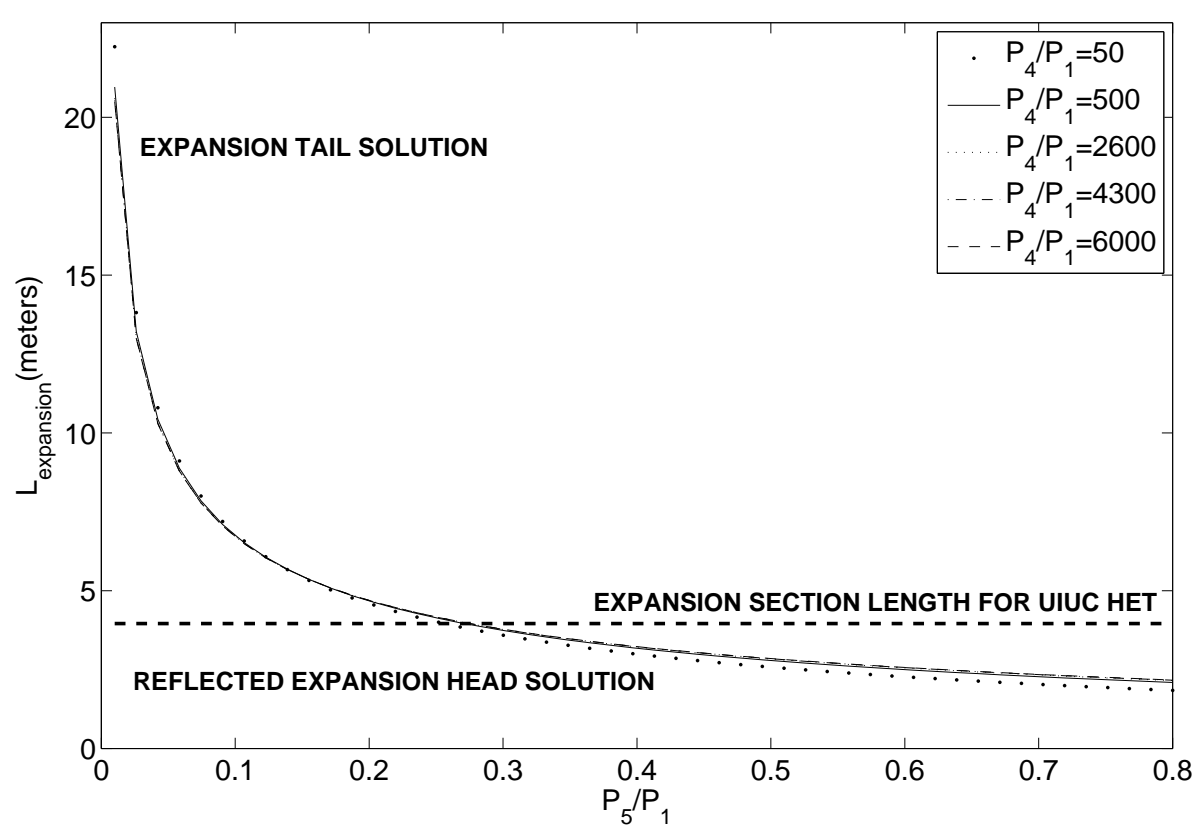

Figure 4. Optimal expansion tube length $L_{\text {expansion }}$ for maximum test time as a function of $\frac{p_{5}}{p_{1}}$ for different $\frac{p_{4}}{p_{1}}$. Air is the test gas in the driven section. The driver and expansion sections are filled with helium.

\section{Design and test time limitations}

Significant test flow disturbances were found to occur in the first expansion tubes. ${ }^{15,16}$ It was seen that the test flow was rendered completely unacceptable due to the presence of high frequency fluctuations. Paull and Stalker theoretically showed that the dominant part of this noise can be modelled as acoustic lateral waves, which can be heavily minimized by decreasing the primary contact surface interface sound speed ratio $\frac{c_{3}}{c_{2}} \cdot{ }^{13}$ An analytical relationship for this sound speed ratio is:

$$
\frac{c_{3}}{c_{2}}=\frac{\left(\frac{p_{4}}{p_{1}}\right)^{\frac{1-2 \gamma_{4}}{2 \gamma_{4}}}\left(\frac{c_{4}}{c_{1}}\right)}{\sqrt{\frac{\frac{2}{M_{s}^{2}}+\gamma_{1}-1}{\gamma_{1}+1}}\left[1+\frac{2 \gamma_{1}\left(M_{s}^{2}-1\right)}{\gamma_{1}+1}\right]^{\frac{1}{2 \gamma_{4}}}}
$$

The shock Mach number $M_{s}$ comes from the implicit shock tube relationship:

$$
\frac{p_{4}}{p_{1}}=\frac{2 \gamma_{1} M_{s}^{2}-\left(\gamma_{1}-1\right)}{\gamma_{1}+1}\left[1-\frac{\gamma_{4}-1}{\gamma_{1}+1}\left(\frac{c_{1}}{c_{4}}\right)\left(M_{s}-\frac{1}{M_{s}}\right)\right]^{\frac{-2 \gamma_{4}}{\gamma_{4}-1}}
$$

For $\gamma_{4}=1.667$ and $\gamma_{1}=1.397$, Figure 5 shows the relationship between the primary contact surface sound speed ratio and $\frac{p_{4}}{p_{1}}$. The result shows that the sound speed ratio and, therefore, 
the test flow disturbance can be reduced by increasing the driver-driven gas pressure ratio. Paull and Stalker do not suggest a value for the sound speed ratio which produces acceptable amounts of noise instead identifying "high enthalpy" and "low enthalpy" conditions as $\frac{c_{3}}{c_{2}}$ less than or greater than one respectively. Low enthalpy conditions were found to be unacceptably noisy. Based on test time pitot data obtained in the current HET facility, it is suggested that the upper limit for $\frac{c_{3}}{c_{2}}$ be set at 0.55 . The $p_{7, p i t o t}$ value is obtained from the pressure trace by averaging over the constant pressure region which follows the arrival of the contact surface (see Figure 16 and Section III B). The noise limit of 0.55 corresponds to the point whereby the fluctuations are at most $\pm 50 \%$ that of the mean value within the test time section of the pitot trace. This value will in turn correspond to a minimum allowable $\frac{p_{4}}{p_{1}}$ value. This value is $\frac{p_{4}}{p_{1}}=1248$ for the current facility. Figure 5 shows the regime in which the HET facility can operate and is bounded by the experimentally observed acceptable sound speed ratio. The upper bound on the pressure ratio is set by the design yield strength of the tube.

The dependence of the test time $t_{\text {test }}$ on $\frac{p_{5}}{p_{1}}$ for different $\frac{p_{4}}{p_{1}}$ values is illustrated in Figure 6 . For a given $\frac{p_{5}}{p_{1}}$ value, test time can be increased by decreasing the $\frac{p_{4}}{p_{1}}$ value. However, there is a limitation placed upon the minimum $\frac{p_{4}}{p_{1}}$ value due to the flow disturbance considerations of the $\frac{c_{3}}{c_{2}}$ ratio as discussed above. The limit for a sound speed ratio of 0.55 is shown as the vertical line in the figure. Acceptable test conditions are to the right of this line. One can see that within this acceptable regime, the dominant influencing factor is that of the $\frac{p_{5}}{p_{1}}$ value rather than the $\frac{p_{4}}{p_{1}}$ value. Thus, the noise limitation tends to dictate the choice of $\frac{p_{4}}{p_{1}}$, rather than test time considerations. For a certain run condition, this vertical line also indicates the maximum allowable test time. A great advantage of expansion tubes is the range of

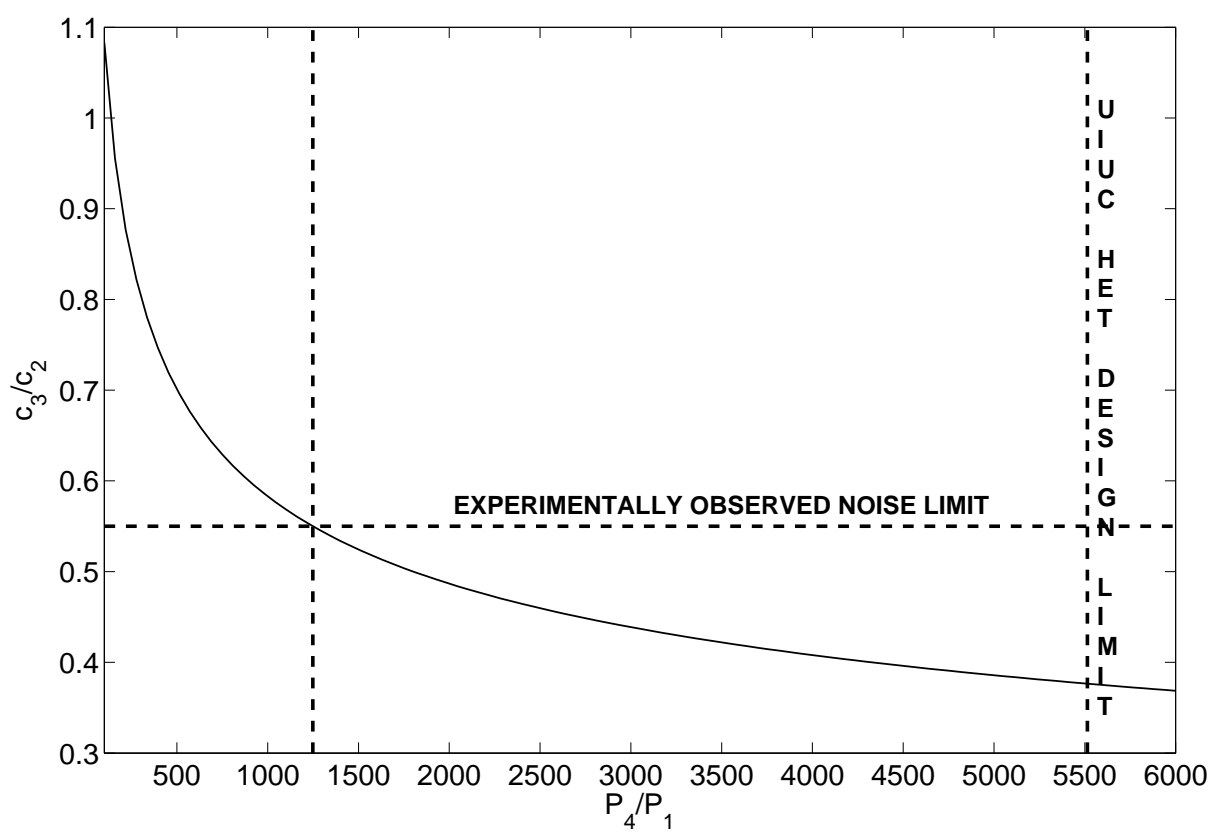

Figure 5. Primary interface sound speed ratio as a function of $\frac{p_{4}}{p_{1}}$. Air is the test gas in the driven section. The driver and expansion sections are filled with helium.

test flow Mach numbers that can be accessed by simply varying the initial pressures. The HET facility has been designed to produce a range of test flows with Mach numbers from 


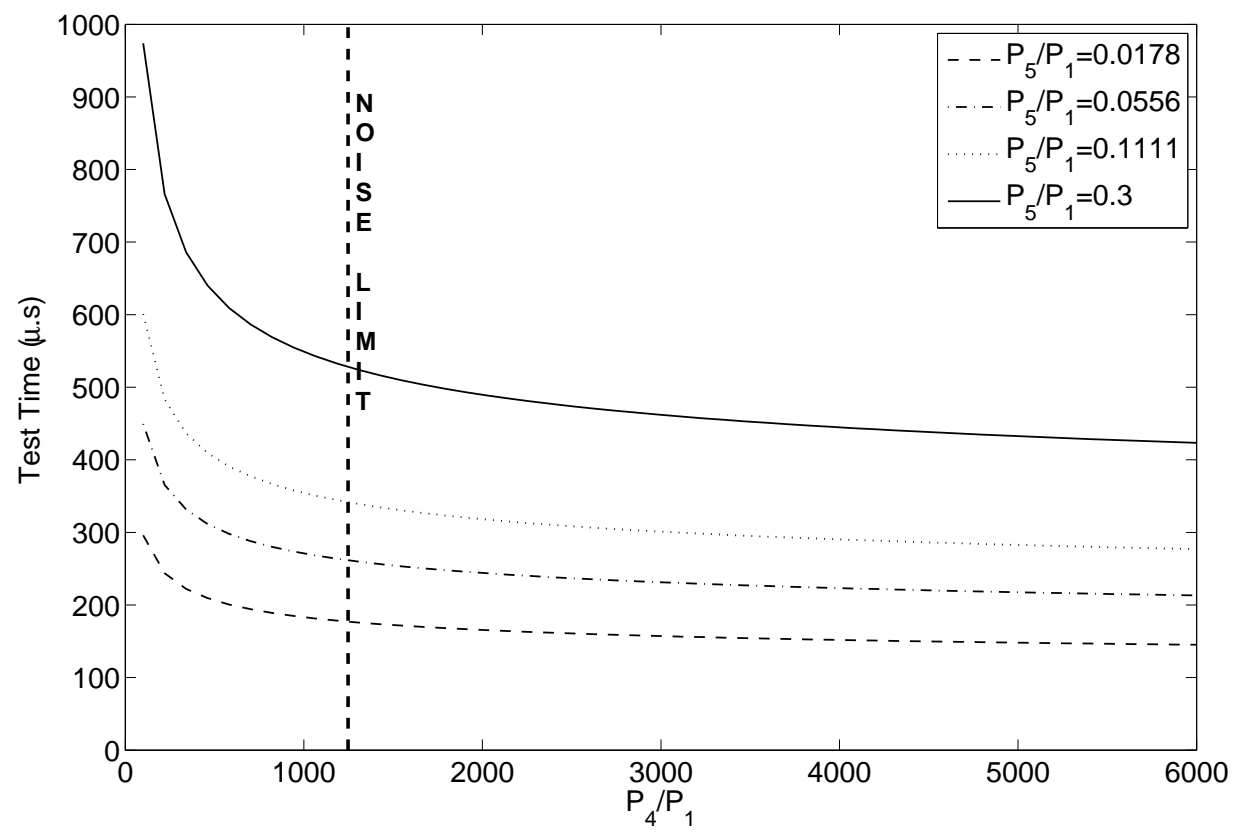

Figure 6. Test time as a function of $\frac{p_{4}}{p_{1}}$ for different $\frac{p_{5}}{p_{1}}$ values. Air is the test gas in the driven section. The driver and expansion sections are filled with helium.

3 to 7.1, as shown in Figure 7. We have selected 3 run conditions for aerodynamic studies, Air-1, Air-2 and Air-3, as summarized in Table 2. The flow Mach number $M_{7}$ can essentially be selected using the expansion-driven pressure ratio $\frac{p_{5}}{p_{1}}$. Figure 7 shows the Mach number is increasing with decreasing $\frac{p_{5}}{p_{1}}$ for all selected values of the driven-driver pressure ratio $\frac{p_{4}}{p_{1}}$. For higher $\frac{p_{4}}{p_{1}}$ ratios, the Mach number is very insensitive to this ratio. Conditions with lower $\frac{p_{4}}{p_{1}}$ ratios exhibit more sensitivity, however, these low pressure ratios may be eliminated as potential run conditions due to noise minimization considerations. It is evident that the dominant factor for both the expansion tube sizing and test section Mach number is the $\frac{p_{5}}{p_{1}}$ ratio. Figure 7 also shows that in order to maintain high Mach number operation for the current facility, $\frac{p_{5}}{p_{1}}$ should be kept below 0.3 .

As previously mentioned, in the results from Figure 6, one can see that in order to increase the test time for a given $\frac{p_{4}}{p_{1}}$ value, the $\frac{p_{5}}{p_{1}}$ ratio must be increased. Figure 7 , however, shows that an increase in $\frac{p_{5}}{p_{1}}$ causes a reduction in $M_{7}$. This demonstrates the need for a working balance within the design process which takes into account the restrictions, dominant factors and fundamental goals of the facility.

So far in this discussion, we have only considered the arrival of the secondary expansion tail or the reflected expansion head as the limiting conditions for the test time. As discussed above, a third possibility is the arrival of the primary expansion wave. As seen in Figure 1, this possibility includes the arrival of the expansion reflected from the driver wall and its tail. The absolute times that the reflected primary driver expansion head, primary driver expansion tail and the second contact surface arrive at the end of the tube are denoted as $t_{r d h}, t_{d e t}$ and $t_{2 c s}$ respectively. A ratio between $t_{r d h}$ and $t_{2 c s}$ and the test time $t_{t e s t}$ for a 
Table 2. Selected theoretical test conditions for the HET facility.

\begin{tabular}{lrrr} 
& Air-1 & Air-2 & Air-3 \\
\hline$M_{7}$ & 7.29 & 5.77 & 4.96 \\
$T_{7}, \mathrm{~K}$ & 740 & 1010 & 1080 \\
$p_{7, p i t o t}, \mathrm{kPa}$ & 67.1 & 90.4 & 117 \\
$p_{67}, \mathrm{kPa}$ & 0.974 & 2.09 & 3.64 \\
$u_{7}, \mathrm{~m} / \mathrm{s}$ & 3980 & 3670 & 3410 \\
$\rho_{7}, \mathrm{~kg} / m^{3}$ & 0.005 & 0.007 & 0.012 \\
Test time, $\mu \mathrm{s}$ & 158 & 225 & 293 \\
$\underline{c_{3}}$ & 0.44 & 0.42 & 0.44 \\
Initial Pressures $^{2}$ & & & \\
Driver Section $^{1}, \mathrm{kPa}$ & 4300 & 4300 & 4300 \\
Driven Section, $\mathrm{kPa}_{\text {Expansion Section, mTorr }}$ & 1.5 & 1.2 & 1.2 \\
Expo & 500 & 1000
\end{tabular}

1. Based on the burst pressure of $0.159 \mathrm{~cm}$ thick Al-5052 diaphragm.

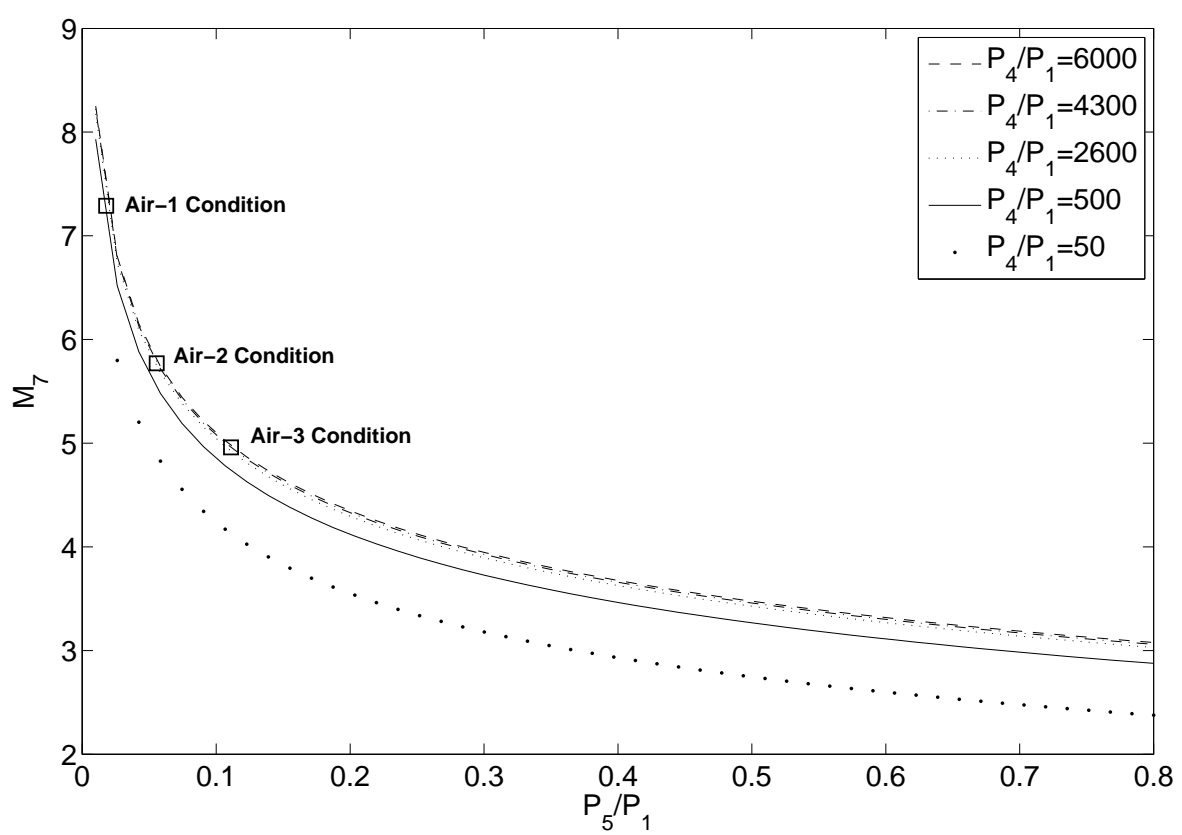

Figure 7. Test gas Mach number $M_{7}$ as a function of $\frac{p_{5}}{p_{1}}$ for different $\frac{p_{4}}{p_{1}}$ values. Air is the test gas in the driven section. The driver and expansion sections are filled with helium. 
certain run condition is given as:

$$
\beta_{3}=\frac{t_{t e s t}}{t_{r d h}-t_{2 c s}}
$$

A ratio between $t_{d e t}$ and $t_{2 c s}$ and the test time is defined as:

$$
\alpha_{3}=\frac{t_{t e s t}}{t_{d e t}-t_{2 c s}}
$$

For the reflected primary driver expansion condition to be considered, the time between the arrival of the second contact surface and the reflected primary driver expansion head should be of the same order as the test time $t_{\text {test }}$, such that $\beta_{3} \sim 1$. The variation of $\beta_{3}$ with $\frac{p_{4}}{p_{1}}$ for four different $\frac{p_{5}}{p_{1}}$ values is shown in Figure 8. As with Figures 5 and 6 , the vertical line represents the noise limitation for $\frac{c_{3}}{c_{2}}=0.55$. Based on the discussion regarding Figure 7 , the $\frac{p_{5}}{p_{1}}$ ratio should be kept below 0.3 . If these two limitations are adhered to then the maximum $\beta_{3}$ is about 0.25 which is significantly less than one, as shown in Figure 8. A similar analysis for the primary driver expansion tail yields that the maximum $\alpha_{3}$ is about 0.13 , which is also significantly less than one. The results for this case are presented in Figure 9. Please note that in both Figure 8 and $9, \beta_{3}$ and $\alpha_{3}$ are plotted on logarithmic scales. Therefore, it is a reasonable conclusion to say that within the current operating regions, the primary expansion wave can be neglected as a test time limitation.

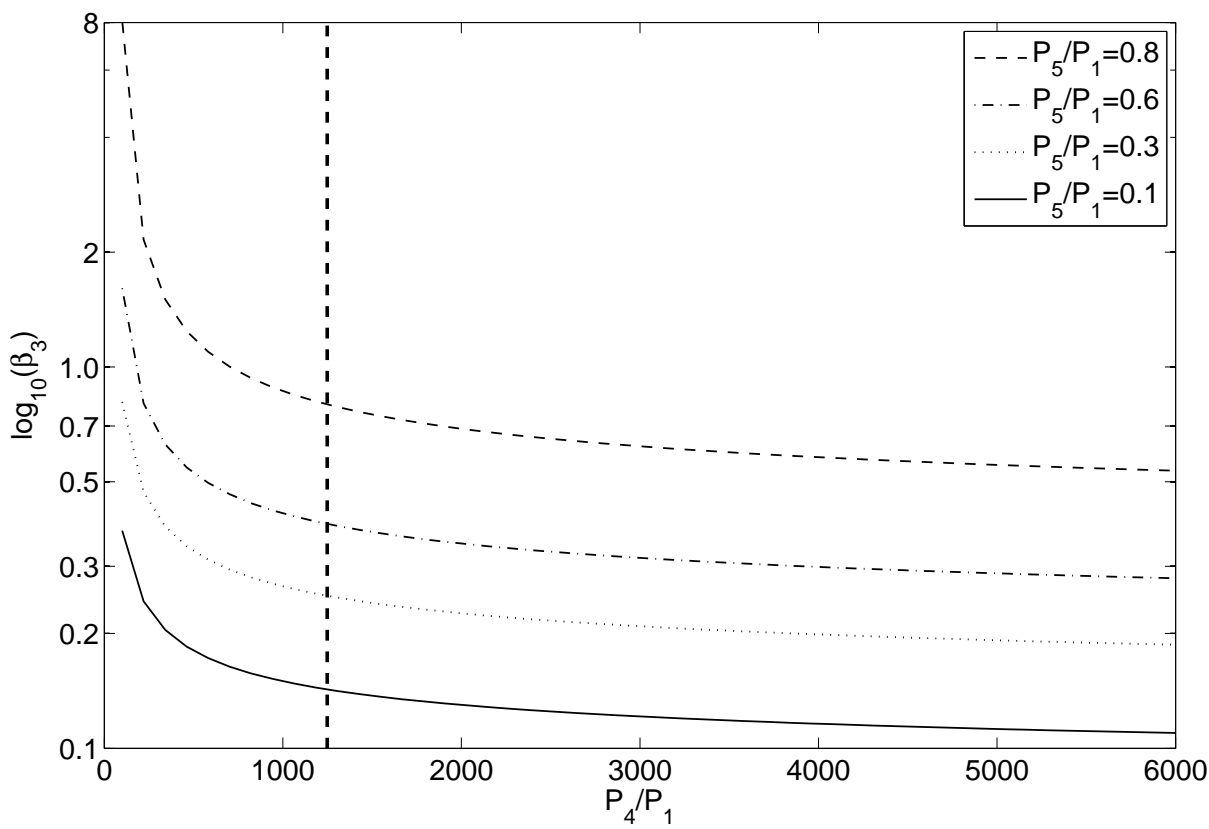

Figure 8. The ratio $\beta_{3}$ as a function of $\frac{p_{4}}{p_{1}}$ for different $\frac{p_{5}}{p_{1}}$ values.

In addition to tube strength considerations, there is also the requirement of having subatmospheric pressure in the facility after each experiment. Impulse facilities such as expansion tubes typically have an O-ring sliding seal between the tube and the test section. If the post-experiment pressure exceeds atmospheric pressure, this can lead to the rupture of the 


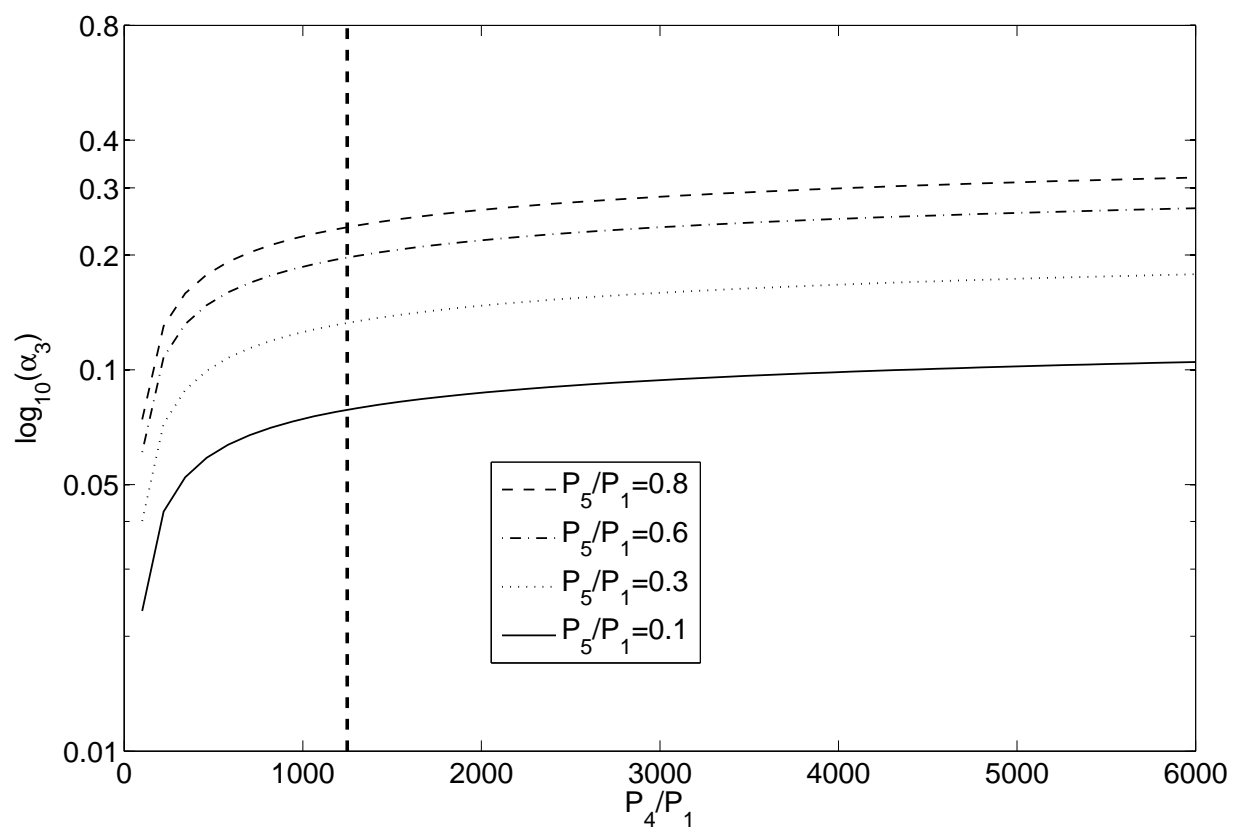

Figure 9. The ratio $\alpha_{3}$ as a function of $\frac{p_{4}}{p_{1}}$ for different $\frac{p_{5}}{p_{1}}$ values.

sliding seal. The post-experiment pressure $p_{\text {final }}$ can be estimated as:

$$
p_{\text {final }}=\frac{p_{4} V_{\text {driver }}+p_{1} V_{\text {driven }}+p_{5} V_{\text {expansion }}}{V_{\text {total }}}
$$

where $V_{\text {driver }}, V_{\text {driven }}$ and $V_{\text {expansion }}$ are the volumes of the driver, driven and expansion sections respectively. $V_{\text {total }}$ is the total volume of the entire facility and $V_{\text {expansion }}$ includes both the volume of the dump tank and the expansion tube section. This criterion can be used to determine the required dump tank volume if the tube dimensions have already been selected. Performing an order of magnitude analysis for the HET facility under current operating condition ranges, yields a simplified limit upon the maximum allowable $p_{4}$ :

$$
p_{4}<\frac{p_{a t m} V_{\text {total }}}{V_{\text {driver }}}
$$

where $p_{a t m}$ is the atmospheric pressure. It is suggested, however, that a reasonable factor of safety is used in the above equation. The current HET design maintains a final facility pressure below $0.85 \mathrm{~atm}$ (factor of safety $=1.17$ ).

\section{HET facility design and operation}

The HET facility is constructed of honed (0.2 $\mu \mathrm{m}$ Ra) 304/304L stainless steel with an inner diameter of $152 \mathrm{~mm}$ and a wall thickness of $0.95 \mathrm{~cm}$. Tube lengths were chosen based on the theoretical gas dynamic calculations described in the preceeding sections. The length of the driver section is $1.22 \mathrm{~m}$, while the driven and expansion sections are both $3.96 \mathrm{~m}$ long. The tube will withstand initial pressures up to $5500 \mathrm{kPa}$ and the facility is capable of 
a vacuum of less than 100 mTorr. The facility is mounted on linear bearings for easy access to the diaphragm stations and to allow tube recoil during the experiment. A schematic of the HET and a picture of the completed facility are both shown in Figures 10 and 11 respectively. The test section shown in Figure 12 has a sliding seal and four-way $100 \mathrm{~mm}$ diameter optical access. A sting may be mounted from the rear of the test section for pitot probes and models. Ten instrumentation ports are located along the tube, of which four are currently instrumented with piezoelectric pressure transducers for wave time-of-arrival and pressure profile measurements. A more detailed description of the facility design and experimental setup is reported in Dufrene. ${ }^{17}$

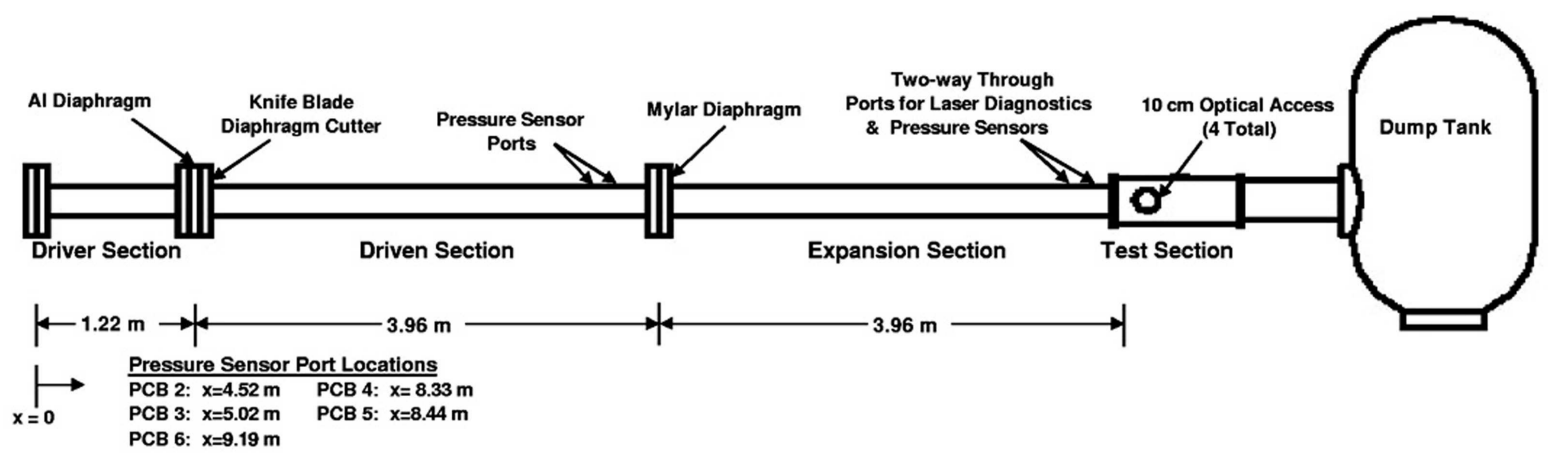

Figure 10. HET schematic. Distances referenced from the inner wall of the driver.

\section{A. Primary and secondary diaphragm selection}

The primary diaphragm is typically $0.159 \mathrm{~cm}$ thick 5052-H32 Al, which has a burst pressure of $4300 \pm 140 \mathrm{kPa}$. Knife blades (Figure 15) were found to produce a more repeatable burst pressure in comparison to scored diaphragms or naturally burst diaphragms. End-mill scored diaphragms resulted in variations of $\pm 1000 \mathrm{kPa}$ in the burst pressure. In addition, petal detachment was commonly observed with both naturally burst and scored diaphragms. The knife blade design was based on GALCIT's 17 -in and 6-in shock tubes. ${ }^{18}$ The knife blades result in a burst pressures reproduciblity of $\pm 140 \mathrm{kPa}$, and the petals always remain intact.

The secondary diaphragm rupture is an extremely important issue in expansion tube operation as non-ideal rupture interferes with the unsteady expansion wave that is used for the secondary acceleration process of the test gas. For this reason, the first set of experiments run in the HET facility were used to determine the best material and thickness for the secondary diaphragm. An extensive range of materials was investigated, including aluminum foil, wax paper, and a variety of plastics. The ideal material is one that breaks cleanly and quickly, yet can sustain the initial pressure difference. Aluminum foil and wax paper could not sustain the required pressure difference. The most promising plastics were Mylar and cellophane.

Diaphragm opening time can be estimated from Eq. 12, where the opening time $t_{0}$ is a function of the material density $\rho$, the petal base length $b$, the petal thickness $\tau$, and the static burst pressure $P .{ }^{19}$ The constant $K$ typically ranges from 0.91 to $0.95 .{ }^{19}$ Petal base length is a function of tube size, and the densities of Mylar and cellophane are very similar. By minimizing thickness and maximizing natural burst pressure, opening time is minimized 


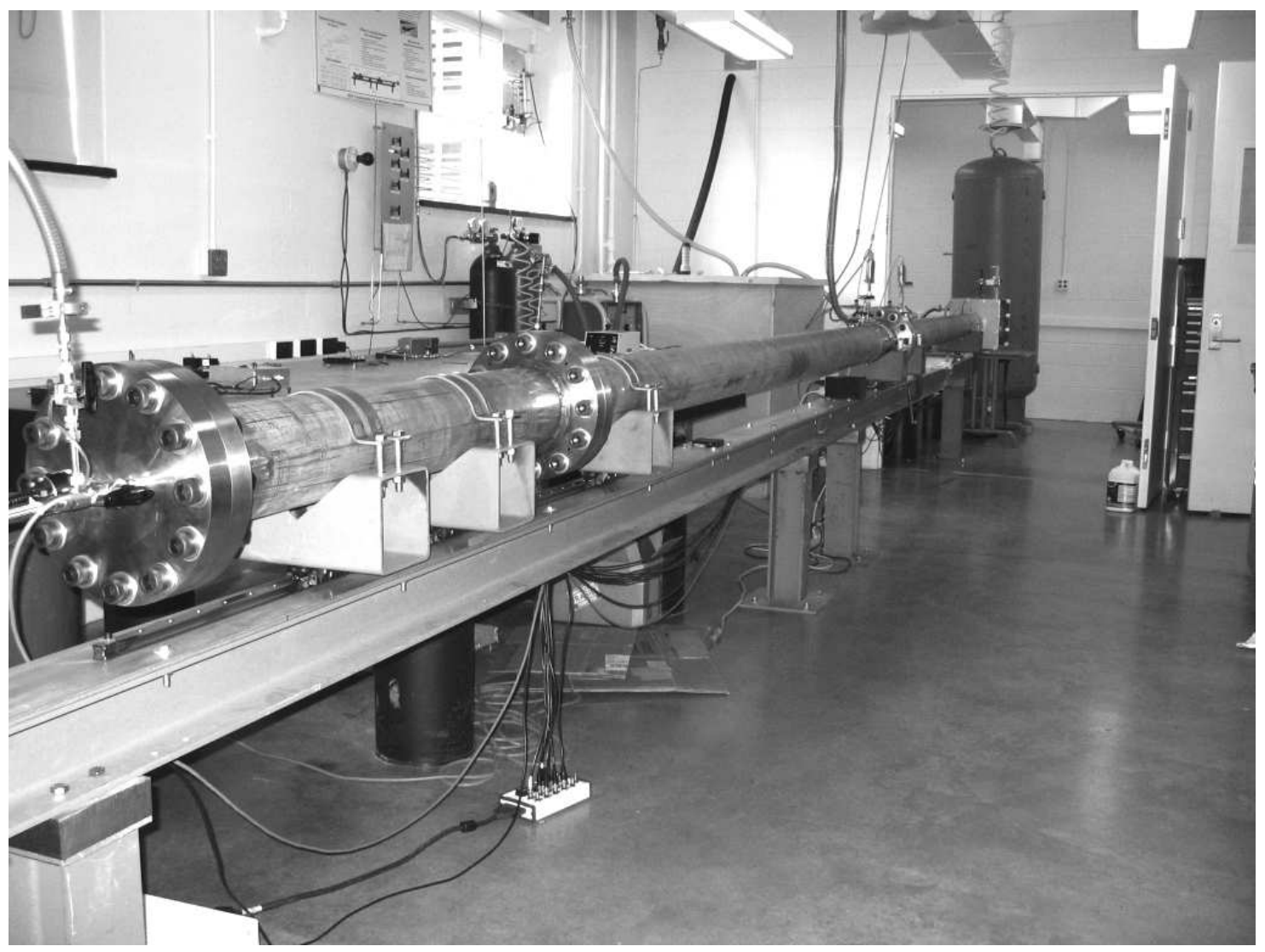

Figure 11. Photograph of HET facility looking downstream.

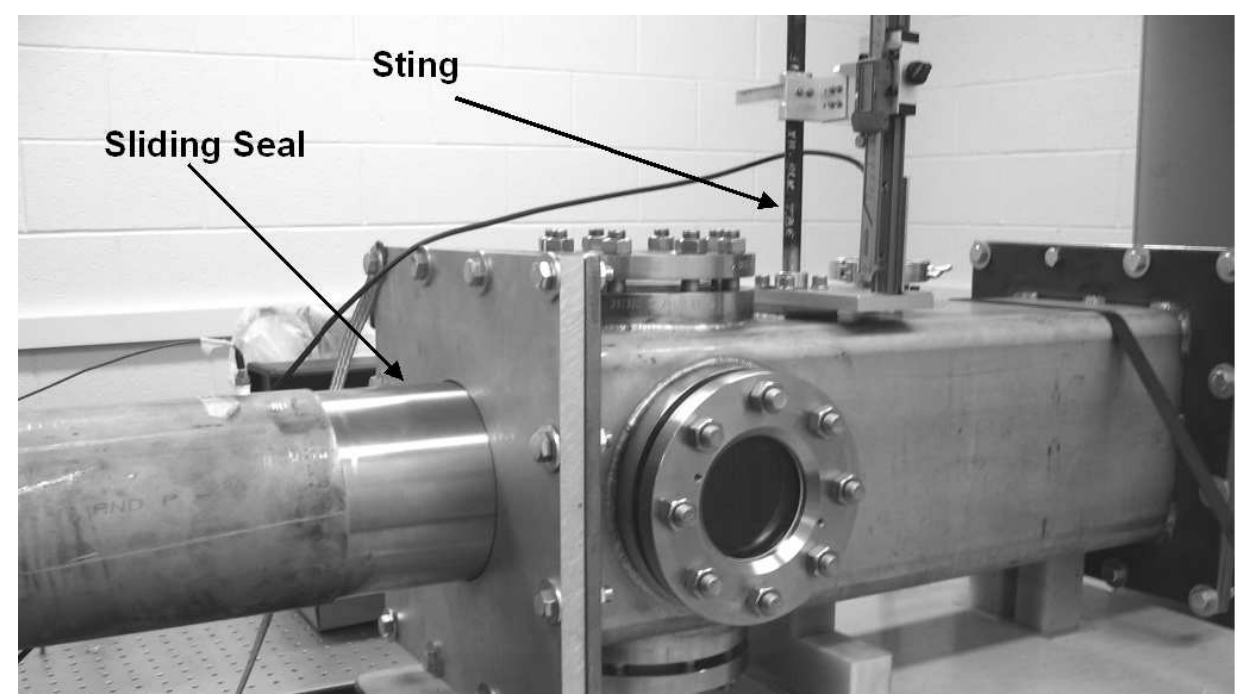

Figure 12. Photograph of test section with sliding seal. 
which is the goal. These two parameters are directly related and are a function of material strength. Since Mylar is much stronger than cellophane, it was expected that Mylar would be the diaphragm of choice. It is important to note that this analysis is simplified, because these diaphragms are subjected to a dynamic rather than a static loading.

$$
t_{0}=K \sqrt{\frac{\rho b \tau}{P}}
$$

In order to determine the best material for the secondary diaphragm, several experiments with low driver pressures were conducted. Pressure traces can be seen Figure 13. The initial pressures for these experiments were $500 \mathrm{kPa}, 10 \mathrm{kPa}$ and $1 \mathrm{kPa}$ in the driver, driven and expansion sections respectively. For the 4th experiment, the driver pressure was doubled. Pressure histories were measured at the third pressure sensor station in the driven section, which was $0.12 \mathrm{~m}$ from the secondary diaphragm, in order to investigate the possibility of a reflected shock from that diaphragm. Under ideal operating conditions, no reflected shock exists. Assuming that the secondary diaphragm is replaced by a hard wall, it would take approximately $100 \mu \mathrm{s}$ for a reflected shock to reach station 3 in these experiments. As seen in Figure 13, it actually takes much longer for the reflected wave to appear in the pressure trace, which is consistent with the flexible boundary that actually exists. From these pressure traces, it was determined that the thinner diaphragms and higher driver pressures should produce operating conditions closer to the ideal scenario. Our results compare well with the results from Tohoku University in Japan. ${ }^{11}$ They built a transparent test section and looked at the development of the reflected shock front for various thicknesses of Mylar and cellophane diaphragms. They report that a $3 \mu \mathrm{m}$ Mylar diaphragm resulted in a reflected shock wave that was regarded as a Mach wave, and at that condition the diaphragm's mechanical influence was practically eliminated. Unfortunately, the two thicknesses of Mylar they used were $25 \mu \mathrm{m}$ and $3 \mu \mathrm{m}$ which are an order of magnitude apart. The secondary diaphragm of choice for HET was found to be $12.7 \mu \mathrm{m}$ Mylar. Heat treated diaphragms were also investigated. The hope was that through a simple heat treatment, the diaphragms would become more brittle and break more rapidly. No significant improvement was found. In fact, the process occasionally produced small holes in the diaphragm allowing expansion section and driven section gases to mix.

A novel secondary diaphragm cutter consisting of a thin wire cross (Figure 14) was mounted downstream of this diaphragm. Prior to the wire cross, sizable pieces of Mylar diaphragm would be found in the test section after a run. With the addition of the wire cross, fragmentation problems have been resolved without appreciably affecting the test flow.

\section{B. Facility operation}

The operation of HET begins with selecting a particular run condition and mounting the diaphragms. The 5052-H32 Al diaphragm separates the driver and driven sections, while the $12.7 \mu \mathrm{m}$ Mylar diaphragm separates the driven and expansion sections. Each section has its own manifold consisting of a gas fill line, a vacuum line and pressure gauges (MKS Piezo+, KJL-205 ). Due to the low initial pressures in the expansion section, it is evacuated to $500 \mathrm{mTorr}$ or less and then flushed with $10 \mathrm{kPa}$ helium before evacuation to its operating pressure. The driven and expansion sections must be evacuated simultaneously ensuring that 


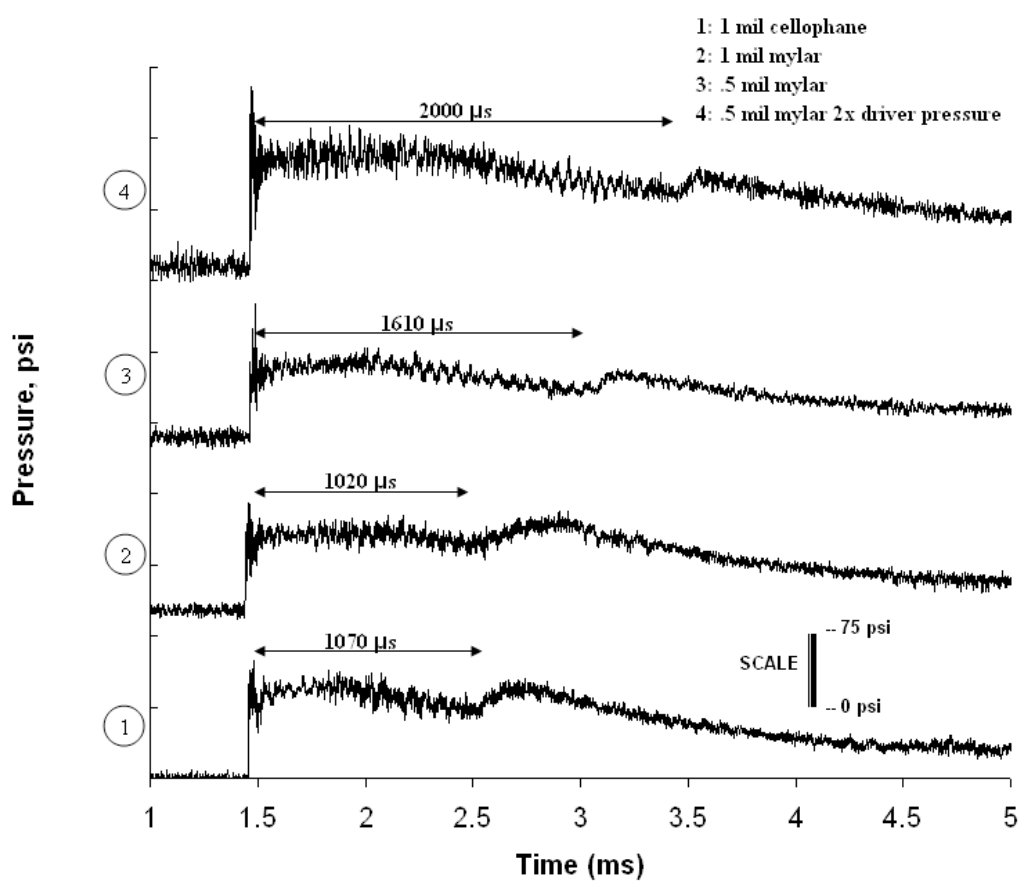

Figure 13. Pressure traces at station 3 for various diaphragms. Four separate experiments were carried out. Traces have been normalized by $150 \mathrm{psi}(1034 \mathrm{kPa})$ for visualization purposes.

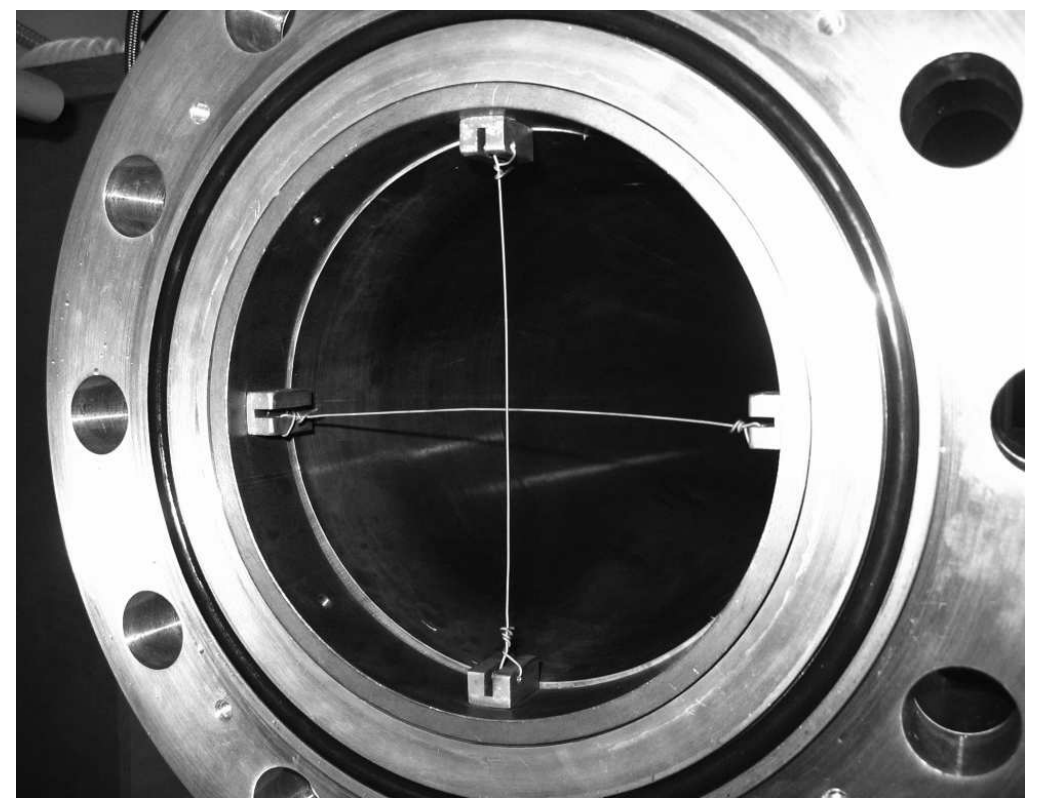

Figure 14. Wire cross downstream of secondary diaphragm. 


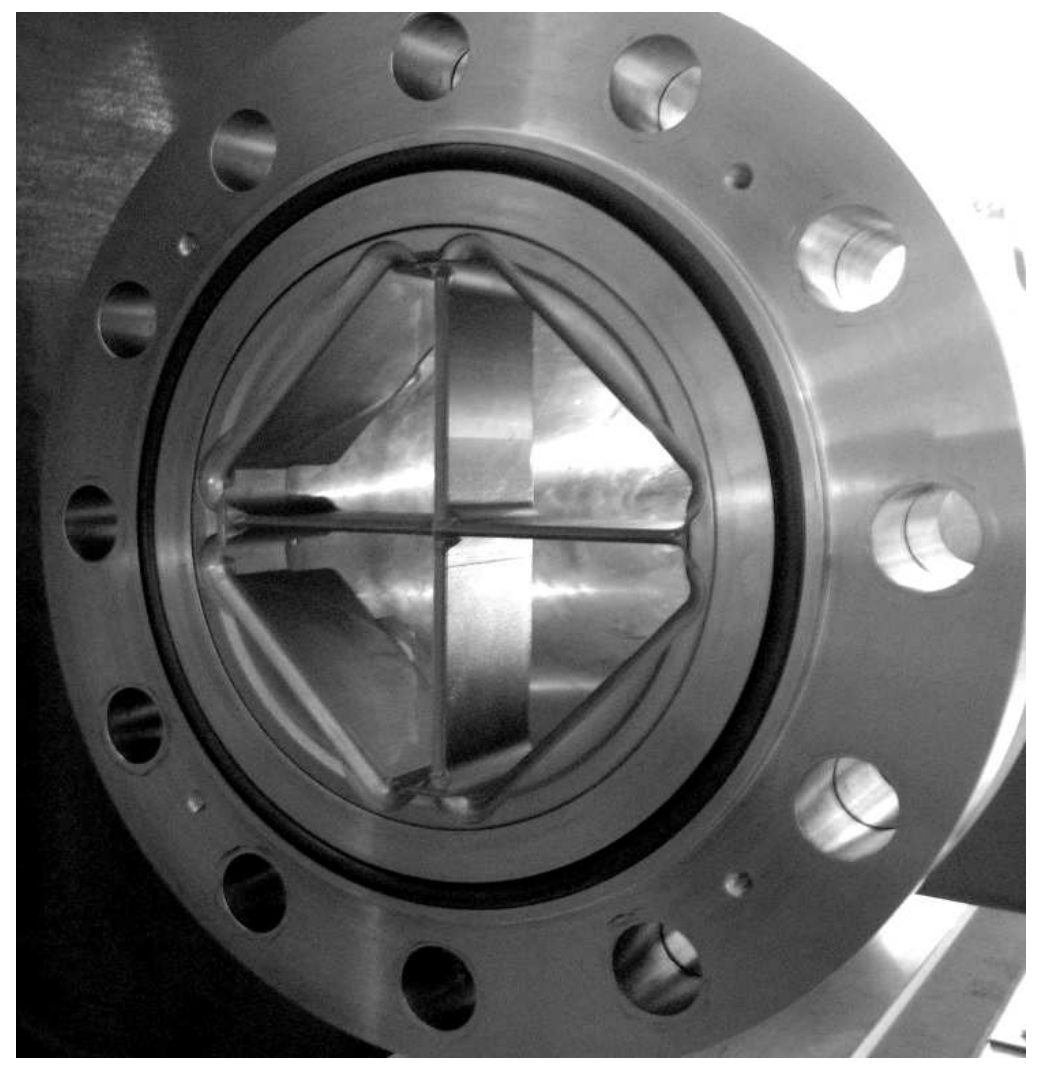

Figure 15. Knife blades at primary diaphragm location.

the pressure difference between the two sections does not exceed $20 \mathrm{kPa}$, to avoid premature rupture of the secondary diaphragm. Once the driven and expansion sections are at the correct pressures, the driver section is evacuated and then pressurized until the primary diaphragm bursts.

A National Instruments modular data acquisition (DAQ) system is used to trigger and collect experimental data. Two dynamic pressure sensors (PCB 113A26) are located in both the driven and expansion sections. These sensors provide pressure and time of arrival data. The sensitivity of these gauges is rated at $\pm 1.45 \mathrm{mV} / \mathrm{kPa}$. In addition to these sensors, a dynamic sensor can be mounted normal to the flow in the test section providing pitot pressure data. A static pressure sensor (Setra 205-2) is located in the driver section to measure the driver pressure before the primary diaphragm ruptures. The accuracy of this gauge is rated at $\pm 27 \mathrm{kPa}$.

A typical pitot trace with air as the test gas and helium as the accelerator gas is shown in Figure 16. From the pitot pressure, the test gas Mach number $M_{7}$ can be calculated assuming the pressure recorded is the stagnation pressure behind a normal shock. Figure 16 corresponds to a Mach number of 7.1 at the Air-1 run condition. The arrival of the accelerator gas and test gas may be identified as indicated in the figure. The shielded transducers have a minimum response time of $13 \mu$ s according to Sutcliffe. ${ }^{20}$ In addition, some test time is lost due to the finite extent of the contact surface. For the example in Figure 16, the time from the arrival of the contact surface to the beginning of the constant pressure test gas is $5 \mu \mathrm{s}$. At this time it is unclear how much of the test time is actually lost to the contact 
surface compared to the response time of the shielded transducer. Depending on whether

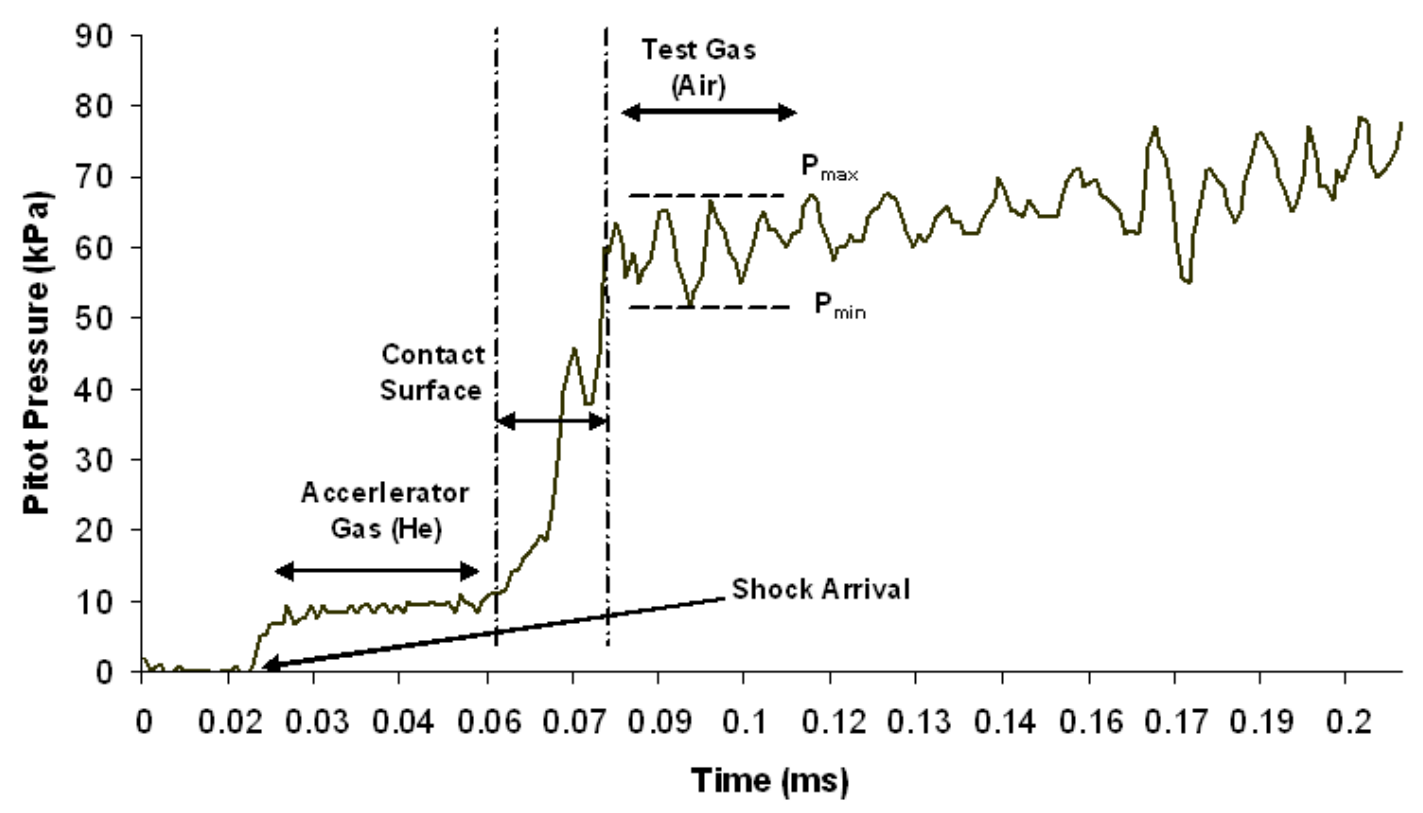

Figure 16. Typical pitot trace for $M=7.1$ (Air-1 run condition).

the test time is limited by the arrival of the reflected expansion head or the expansion tail (as discussed in Section II), the pressure will either rise or drop after the test time. If the waves arrive around the same time (the optimal condition), the pressure variations tend to cancel each other out and look like typical test time noise. Future experiments will focus on alternative diagnostics for contact surface arrival measurements.

\section{Experimental characterization}

Table 3 presents the comparision of experimental data with theoretical predictions for the three run conditions described in Table 2. Pressure transducers located along the tube wall were used for time-of-arrival data to calculate the primary shock velocity. Pitot pressure measurements were made using a dynamic pressure sensor (PCB 113A26) sting-mounted along the centerline of tube, normal to the flow field in the test section. The experimental test time can only be given as an estimate due to reasons discussed in Section III. Shock time-of-arrival data were obtained from pressure transducers mounted along the driven and accelerator sections, however the post-shock pressure in the accelerator section could not be determined because the signal to noise ratio was too poor. Therefore, the experimental Mach number must be calculated using the theoretical prediction of the dynamic pressure within the test section $\left(p_{67}\right)$ along with the experimentally observed $p_{7, p i t o t}$ value.

\section{A. Primary Shock Speed, $u_{s}$}

It can be seen in Table 3 that the experimental shock speed is consistently measured to be 2 to $5 \%$ below the theoretical prediction. Experimental results from other expansion tube 
Table 3. HET facility performance: Experimental measurements (Exp.) vs. Theoretical Prediction (Theory).

\begin{tabular}{|l|rr|rr|rr|}
\hline & \multicolumn{2}{|c|}{ Air-1 } & \multicolumn{2}{|c|}{ Air-2 } & \multicolumn{2}{c|}{ Air-3 } \\
& Theory & Exp. & Theory & Exp. & Theory & Exp. \\
\hline$M_{7}$ & 7.29 & $\mathbf{7 . 1}$ & 5.77 & $\mathbf{5 . 7}$ & 4.96 & $\mathbf{4 . 5}$ \\
$p_{\text {pitot }}, \mathrm{kPa}$ & 67 & $\mathbf{6 4}$ & 90 & $\mathbf{9 0}$ & 117 & $\mathbf{9 2}$ \\
$u_{s}, \mathrm{~m} / \mathrm{s}$ & 2126 & $\mathbf{2 0 6 9}$ & 2187 & $\mathbf{2 1 4 9}$ & 2186 & $\mathbf{2 1 4 0}$ \\
Test time, $\mu \mathrm{s}$ & 158 & $\mathbf{1 0 0}$ & 225 & $\mathbf{1 5 0}$ & 293 & $\mathbf{9 0}$ \\
\hline
\end{tabular}

facilities indicate that the axial variation of the shock speed velocity rises to a peak value above the theoretical value and then continues to decay below this value. ${ }^{9,21}$ It has been postulated that during diaphragm opening, compression waves coalesce to form a downstream shock, whose end result is to produce a stronger combined shock. ${ }^{21,22}$ Physically, therefore, the experimentally observed results can be explained by the process whereby close to the primary diaphragm, the strengthing "compression" effect is more dominant, however, as the viscous effects become more dominant with increasing downstream distance, the shock velocity attenuates. To account for this axial shock velocity variation, the shock velocity is measured as close as possible to the secondary diaphragm station (tranducers at $\mathrm{x}=5.23 \mathrm{~m}$ and $5.28 \mathrm{~m}$, Figure 10). The results from Table 3 indicate that the pressure transducers are within the attentuation region for all the run conditions considered.

Mechanically-induced noise was initially a problem for the wall-mounted transducers. To overcome this, the PCB mounting fixtures were redesigned to accomodate two O-rings which isolate the fixture from the facility wall. This design considerably improved the signal to noise ratio.

\section{B. Test Gas Pitot Profiles and Traces}

For the nominal $M_{7}=7.29$ case, pitot pressure surveys were made in order to determine the size of the core flow. Surveys were conducted at two positions in the test section $31 \mathrm{~mm}$ and $54 \mathrm{~mm}$ downstream of the tube exit. These pressure surveys are shown in Figures 18 and 19. The error bars in the figures are given by the $P_{\max }$ and $P_{\min }$ values that were experimentally measured within the test time (see Figure 16). Flow disturbances have been minimized by following the guidelines suggested by Paull and Stalker ${ }^{13}$ and described in Section II B. However, significant pressure oscillations are still present in the pitot pressure traces. The measurement technique and methodology both contribute to the introduction of noise. In the present study, the mounting configuration consists of a protective shell to prevent debris from striking the transducer, Figure 17. It has been numerically demonstrated that waves form within this shell and create noise which is not within the flow itself. ${ }^{23}$

From these profiles, it appears that the axial separation has no marked effect upon the core flow size over the distance examined. Table 3 shows the pitot pressures are very close to the theoretical pitot pressures, suggesting that the HET is operating near the theoretical predictions. A centerline pitot pressure measurement of $64 \mathrm{kPa}$ corresponded to a Mach number of 7.1. Figure 20 shows a comparison between two similar Mach number conditions: 


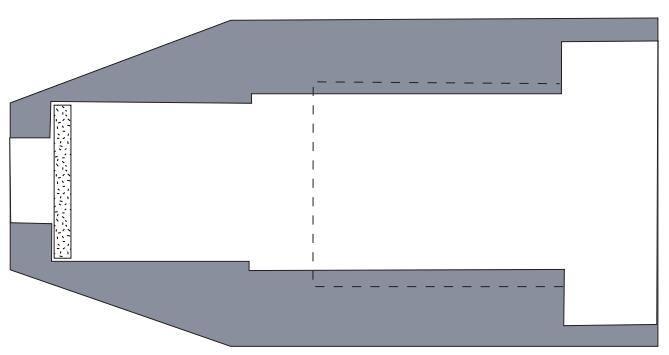

(a)

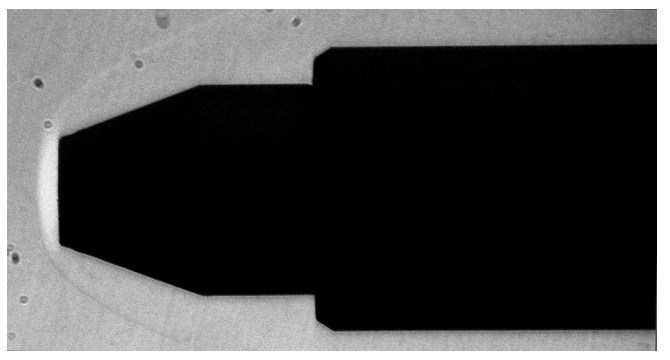

(b)

Figure 17. a) Schematic of pressure transducer mounting for free-stream pitot pressure measurements. A porous grill (with $91 \mathrm{~mm}$ diameter holes) is positioned directly upstream of the transducer diaphragm. b) Schlieren image of flow over the pitot probe mounting.

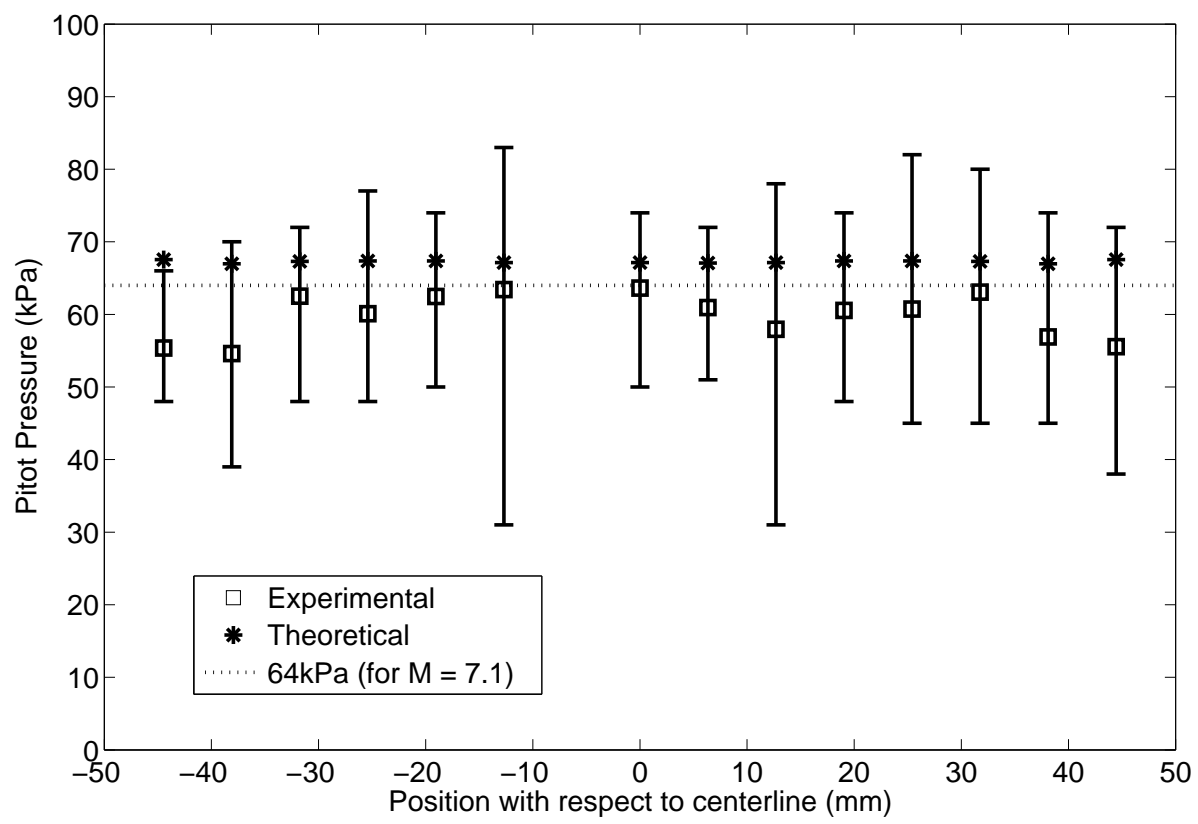

Figure 18. Pitot survey for Air-1 run condition $5.4 \mathrm{~cm}$ downstream from tube exit. 


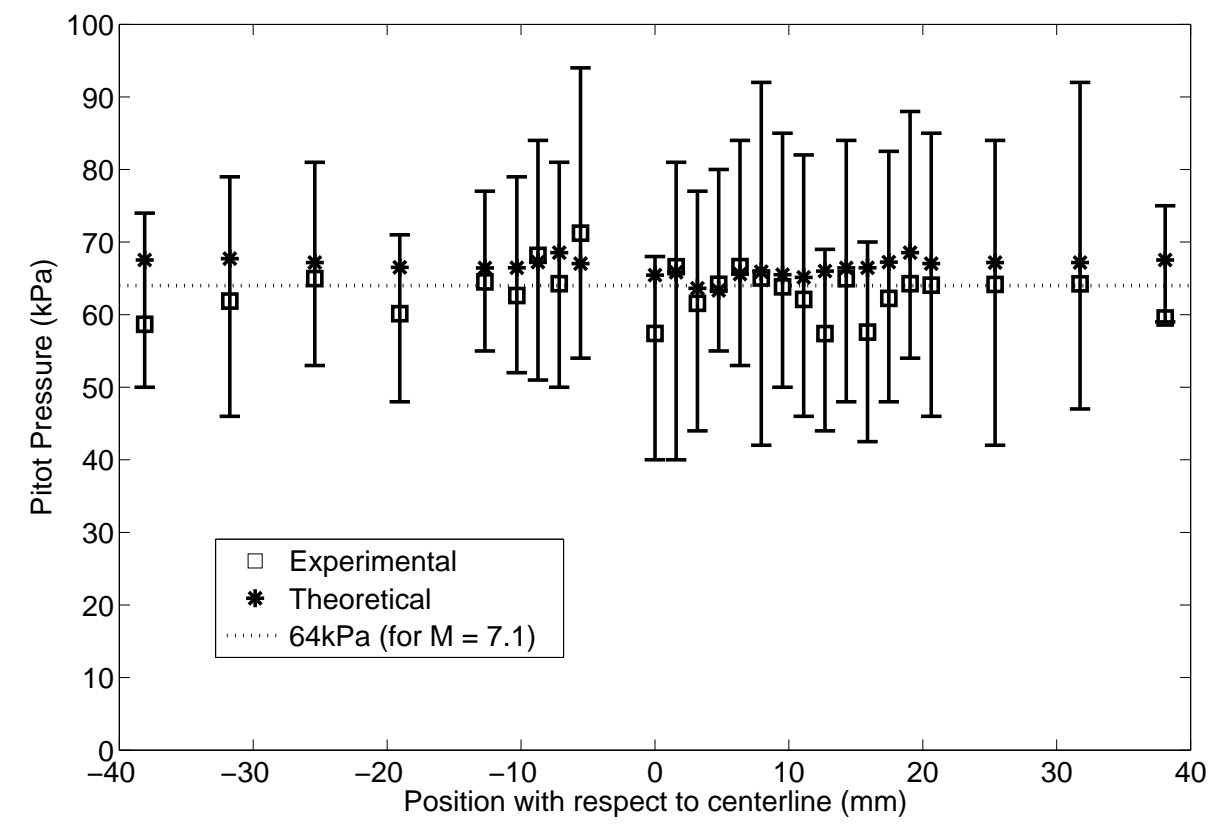

Figure 19. Pitot survey for Air-1 run condition $3.1 \mathrm{~cm}$ downstream from tube exit.

a nominal Mach number 7.20 condition and the Air-1 run condition with nominal Mach number 7.29. The $\frac{c_{3}}{c_{2}}$ ratios for these two cases are 0.74 and 0.44 respectively. Visual inspection confirms the postulation that lowering the $\frac{c_{3}}{c_{2}}$ ratio reduces the noise within the test time and that ratios above 0.55 are unacceptable for the current facility.

As previously mentioned, Paull and Stalker showed that significant test flow noise is caused by the presence of lateral waves and a lower primary interface sound speed ratio impedes this disturbance transmission from the driver gas. They postulated that these lateral waves first appear in the driver gas, are transmitted in the test gas and result in unsteady disturbances once the test gas has been expanded. ${ }^{13}$ They demonstrated that the noise upstream of an unsteady expansion, such as that occuring at the secondary diaphragm, would be focused to one particular frequency provided that a significant drop in the primary interface sound speed ratio occured across the expansion. Therefore, one would expect significantly more frequency focusing at lower sound speed ratios. Figure 20 confirms this, as the Air-1 run condition exhibits a structured test time disturbance whereas the disturbance in the Mach 7.2 case is much less structured.

\section{Flow over wedge geometries}

Schlieren images were taken of the flow over wedge geometries in order to infer the Mach number from the shock angle. The theoretical shock angle $\beta$ is given by the well known $\theta-\beta-M$ relationship, where $\theta$ is the wedge angle:

$$
\tan \theta=2 \cot \beta\left[\frac{M_{7}^{2} \sin ^{2} \beta-1}{M_{7}^{2}\left(\gamma_{7}+\cos 2 \beta\right)+2}\right]
$$




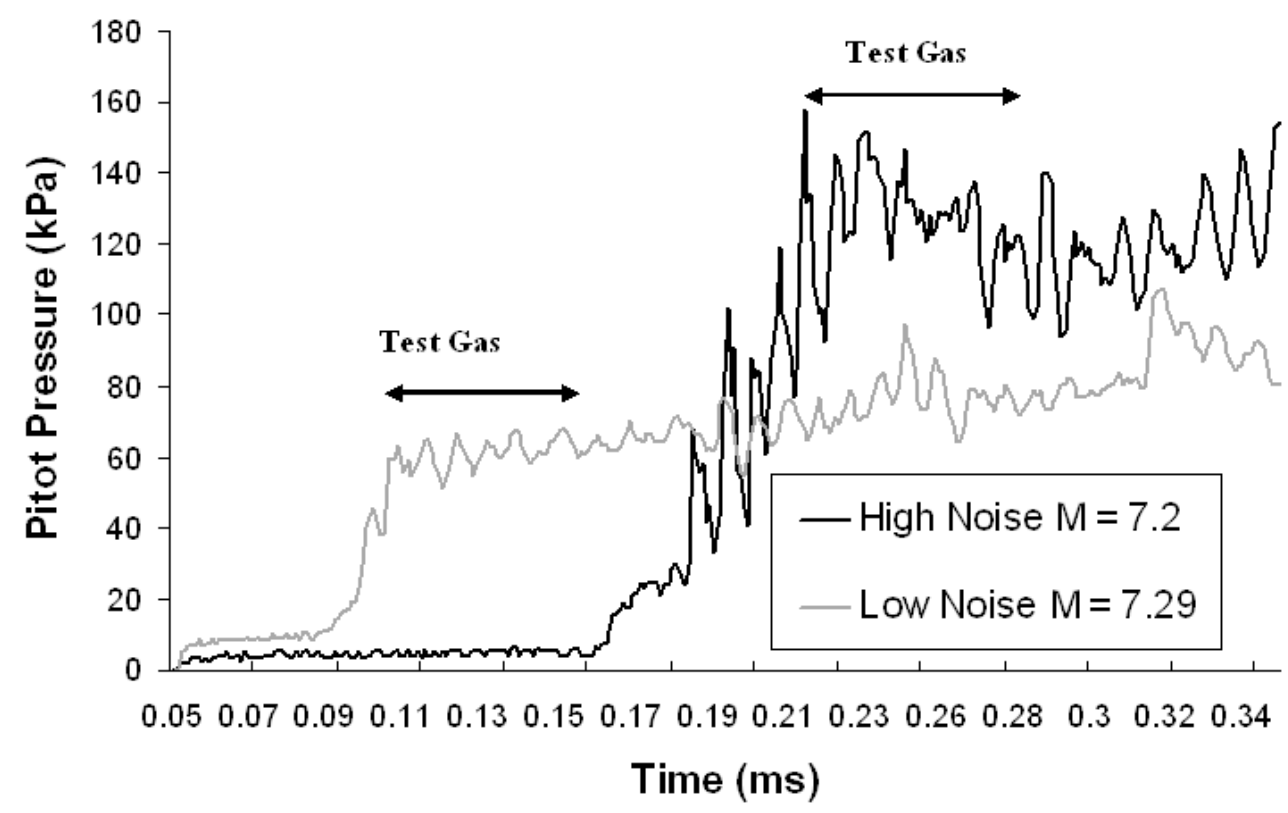

Figure 20. Pitot histories for Mach 7.29 and Mach 7.2. $\frac{c_{3}}{c_{2}}$ values are 0.44 and 0.74 respectively. The Mach 7.2 condition had initial pressures of $p_{4}=4300 \mathrm{kPa}, p_{1}=10 \mathrm{kPa}$ and $p_{5}=500 \mathrm{mTorr}$. The Mach 7.29 condition is the Air-1 condition from Table 2.

Figure 21 shows an image taken at the Air-1 run condition. According to pitot data, the experimental test Mach number was 7.1 and the measured shock angle was 33.65 degrees. From Equation 13, the shock angle should be 33.12 degrees based on a nominal perfect gas Mach Number of 7.29 or 33.23 degrees based on the experimentally measured Mach number of 7.1. The measured shock angle of 33.65 degrees corresponds to a flow Mach number of 6.57. There was a $\pm 0.8 \%$ error in measuring the shock angle.

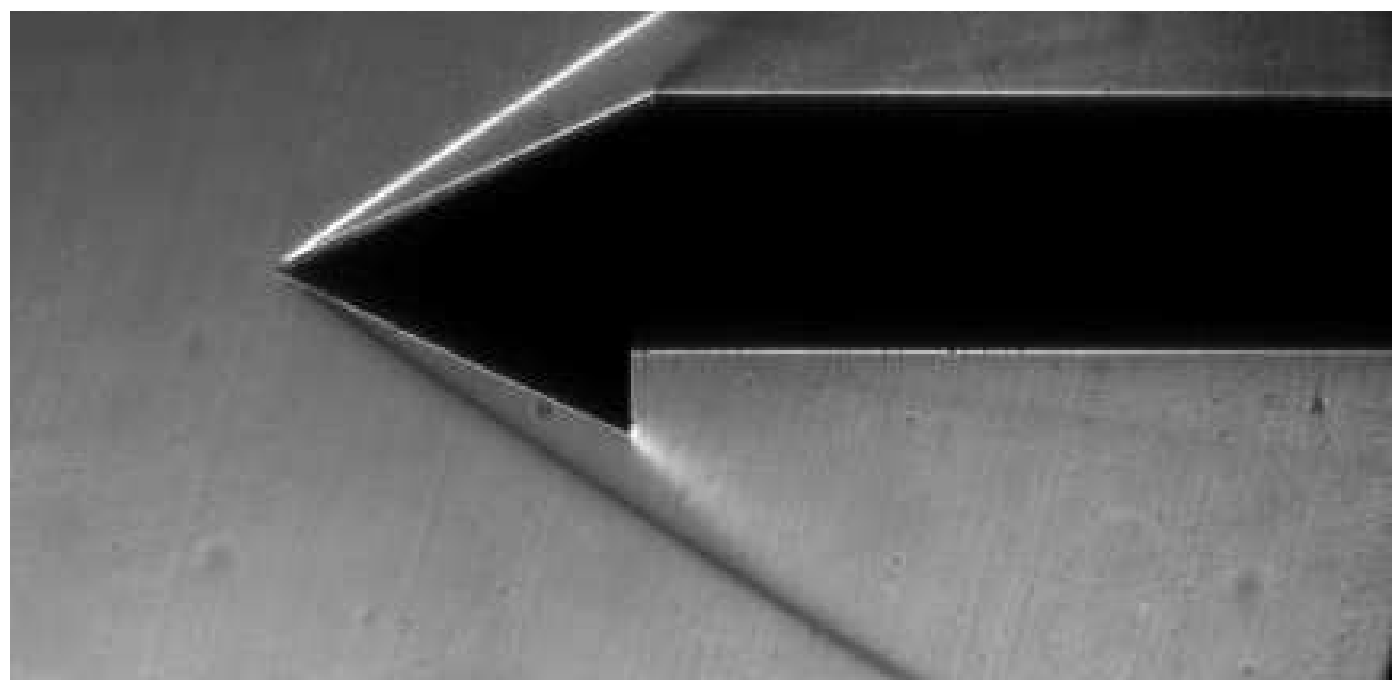

Figure 21. Schlieren image of a 25 degree wedge at Air-1 run condition 


\section{Conclusions}

Expansion tubes have the potential to offer a wide range of high stagnation enthalpy test flow conditions for aerodynamic studies. This range of test conditions is in practice limited by insufficient test time and core flow size, or substantial flow disturbances. ${ }^{9,13}$ A generalized design strategy for small-scale expansion tubes is presented. The procedure was used to design an expansion tube facility capable of producing a range of reasonable quality test flows with Mach numbers over the range 3 to 7.1 .

Gas dynamic calculations of expansion tube operation show that the maximum test time for high enthalpy operating conditions (defined as a expanded driver gas to driven gas sound speed ratio of less than one by Paull and Stalker ${ }^{13}$ ) is obtained when the tail of the expansion wave and the leading characteristic of the reflected expansion coincide. This criterion is used in designing the length of the expansion section. An analytical solution for the passage of the first reflected characteristic through the secondary expansion is presented. We find the interaction of the two expansion waves significantly delays the arrival of the leading characteristic, and accounting for this effect may increase the theoretical test time by as much as $35 \%$.

Large amplitude disturbances contaminated the test flow of the first expansion tubes. ${ }^{15,16}$ Paull and Stalker ${ }^{13}$ found lateral acoustic waves generated in the driver section were a significant source of these disturbances. The penetration of acoustic waves into the test gas could be minimized by minimizing the expanded driver gas to driven gas sound speed ratio. We use Paull and Stalker's criterion to achieve reasonable quality test flow in the current facility. We find that the sound speed ratio $\frac{c_{3}}{c_{2}}$ should be less than 0.55 to reduce the measured pitot pressure peak to peak fluctations to less than $50 \%$ in our facility. The quality of the core flow is expected to be somewhat better than this value, as the shroud and cavity mounting used to protect the transducers in this measurement is expected to contribute to the observed pressure oscillations.

Within these limits, the test flow Mach number and maximum test time can be selected by varying the initial pressures in each of the three sections of the facility. The test flow Mach number $\mathrm{M}_{7}$ is a relatively strong function of the accelerator to driven section pressure ratio $\mathrm{p}_{5} / \mathrm{p}_{1}$, particularly for higher Mach numbers (greater than about 4) which correspond to $\mathrm{p}_{5} / \mathrm{p}_{1}$ values less than about $0.2 . \mathrm{M}_{7}$ is only weakly dependent on the driver to driven gas pressure ratio $\mathrm{p}_{4} / \mathrm{p}_{1}$ for the conditions considered in this study. The test time is also relatively independent of the driver to driven gas pressure ratio for $\mathrm{p}_{4} / \mathrm{p}_{1}$ greater than about 1000 . Since values of $\mathrm{p}_{4} / \mathrm{p}_{1}$ less than 1000 correspond to sound speed ratios with large amplitude flow disturbances, the test time is selected predominately by selecting the accelerator to driven section pressure ratio $\mathrm{p}_{5} / \mathrm{p}_{1}$. Decreasing the $\mathrm{p}_{5} / \mathrm{p}_{1}$ ratio increases the test time, however, the test flow Mach number is also decreased. Thus, the optimal initial pressures can be selected based on specific experimental requirements.

The procedure above was used to design the hypervelocity expansion tube (HET). The HET consists of three sections: a $1.22 \mathrm{~m}$ driver, $3.96 \mathrm{~m}$ driven tube and a $3.96 \mathrm{~m}$ expansion tube, all of honed stainless steel tubing with an inner diameter of $152 \mathrm{~mm}$. The exit of the accelerator tube is connected to a test section with four-way $100 \mathrm{~mm}$ diameter optical ports via a sliding seal. The HET facility is fully operational and capable of being run multiple times per day.

A knife blade cross is used to burst the primary diaphragm. The knife blades have a 
much more reproducible burst pressure of $\pm 140 \mathrm{kPa}$ compared to scored diaphragms which varied $\pm 1000 \mathrm{kPa}$. The knife blades also achieve higher burst pressures than the scored diaphragms without the loss of diaphragm petals.

The influence of the secondary diaphragm is an important issue for expansion tubes. The diaphragm opening time will reduce the available test time and affect the unsteady expansion fan. Flow disturbances may be introduced, and fragmentation may also be an issue. Pressure data was used to investigate the effect of several secondary diaphragm materials and thicknesses on the quality of the test flow. $12.7 \mu \mathrm{m}$ Mylar was selected for all of the current run conditions. A novel crossed wire arrangement was found to result in repeatable test conditions and reduced diaphragm fragmentation without adding appreciable disturbances to the test flow.

A range of test conditions (Mach 3 to 7.1 ) have been verified through pitot pressure measurements and shock angle measurements over simple geometries. Experimental conditions are compared with gas dynamic calculations and found to be in good agreement. Pitot pressure surveys at two downstream locations (31 and $54 \mathrm{~mm}$ downstream of the tube exit) were used to measure the core flow size. The flow is uniform over about $60 \mathrm{~mm}$ for the Mach 7.1 test condition.

\section{Acknowledgments}

This research was funded in part through a Multi-University Research Initiative (MURI) granted by the Air Force Office of Scientific Research (AFOSR), with Dr John Schmisseur as Technical Monitor. Special thanks to Matthew D. Parker for test section design and construction, and data acquisition system installation, to Matt McGilvray for the design of the transducer mounts, and to Dr. Richard Morgan for helpful discussions.

\section{References}

${ }^{1}$ Lukasiewicz, J., Experimental Methods of Hypersonics, Marcel Dekker, Inc., New York, 1973.

${ }^{2}$ Hornung, H., "Experimental Hypervelocity Flow simulation, needs, achievements and limitations," Proc. 1st Pacific Int. Conf. on Aerospace Science and Technology, 1994. 1992

${ }^{3}$ Hornung, H., "Performance data of the new free-piston shock tunnel at GALCIT," AIAA 92-3943,

${ }^{4}$ Albrechcinski, T., Boyer, D., Chadwick, K., and Lordi, J., "Calspan's upgraded 96-inch hypersonic shock tunnel: Its development and application in performance of research and testing at higher enthalpies," AIAA Paper 95-0236, 1995.

${ }^{5}$ Erdos, J., Bakos, R., Castrogiovanni, A., and Rogers, R., "Dual mode Shock Expansion/Reflected Shock Tunnel," AIAA 97-0560, 1997.

${ }^{6}$ Neely, A. J. and Morgan, R. G., "The Superorbital Expansion Tube concept, experiment and analysis," Aeronautical Journal, Vol. 973, 1994, pp. 97-105.

${ }^{7}$ Ben-Yakar, A. and Hanson, R. K., "Characterization of Expansion Tube Flows for Hypervelocity Combustion Studies," Journal of Propulsion and Power, Vol. 18, No. 4, 1992, pp. 943-952.

${ }^{8}$ Trimpi, R. L., "A preliminary theoretical study of the expansion tube, a new device for producing high enthalpy short-duration hypersonic gas flows," Tech. Rep. TR R-133, NASA, 1962.

${ }^{9}$ Shinn, J. L. and Miller, C. G., "Experimental Perfect Gas Study of Expansion-Tube Flow Characteristics," NASA TP 1317, 1978. 
${ }^{10}$ Wegener, M., Sutcliffe, M., and Morgan, R., "Optical study of a light diaphragm rupture process in an expansion tube," Shock Waves, Vol. 10, 2000, pp. 167-178.

${ }^{11}$ Furukawa, T., Aochi, T., and Sasoh, A., "Expansion tube operation with thin secondary diaphragm," 44th AIAA Aerospace Sciences Meeting and Exhibit, Reno, Nevada, Vol. AIAA 2006-1097, 2006.

${ }^{12}$ Daru, V. and Damion, J., "Analysis of flow perturbations in a shock tube due to curvature of the diaphragm," 19th International Symposium of Shock Waves, 1995, pp. 451-456.

${ }^{13}$ Paull, A. and Stalker, R. J., "Test flow distrubances in an expansion tube," Journal of Fluid Mechanics, Vol. 245, 1992, pp. 493-521.

${ }^{14}$ Wintenberger, E., Austin, J. M., Cooper, M., Jackson, S., and Shepherd, J. E., "An Analytical Model for the Impulse of a Single-Cycle Pulse Detonation Engine," J. Propul. Power, Vol. 19, No. 1, 2003 , pp. 22-38.

${ }^{15}$ Spurk, J., "Design, operation and preliminary results of the BRL expansion tube," Fourth Hypervelocity Technical Symposium, A.E.D.C, 1965, pp. 114-144.

${ }^{16}$ Norfleet, G., Lacey, J., and Whitfield, J., "Results of an experimental investigation of the performance of an expansion tube," Fourth Hypervelocity Technical Symposium, A.E.D.C, 1965, pp. 49-110.

${ }^{17}$ Dufrene, A., "Design and Characterization of the Hypervelocity Expansion Tube," Tech. rep., M.S. thesis, University of Illinois at Urbana-Champaign, 2006.

${ }^{18}$ Roshko, A. and Baganoff, D., "A Novel Device for Bursting Shock-Tube Diaphragms," Physics of Fluids, Vol. 4, 1961, pp. 1445-1446.

${ }^{19}$ Rothkopf, E. M. and Low, W., "Diaphragm opening process in shock tubes," Physics of Fluids, Vol. 17, No. 6, 1974, pp. 1169-1173.

${ }^{20}$ Sutcliffe, M. A. and Morgan, R. G., "The measurement of Pitot pressure in high enthalpy expansion tubes," Measurement Science and Technology, Vol. 12, 2001, pp. 327-334.

${ }^{21}$ Ikui, T., Matsuo, K., and Nagai, M., "Investigations of the Aerodynamic Characteristics of the Shock Tubes (Part 2, On the Formation of Shock Waves)," JSME Bulletin, Vol. 12, No. 52, 1969, pp. 783-792.

${ }^{22}$ White, D., "Influence of Diaphragm Opening Time on Shock-Tube Flows," Journal of Fluid Mechanics, Vol. 4, No. 6, 1958, pp. 585-599.

${ }^{23}$ Buttsworth, D. and Jacobs, P., "Measurement of fluctuations in a Mach 4 shock tunnel nozzle flow," In 7th Australasian Heat and Mass Transfer Conference, July, 2000. 